\title{
A continuum mechanics based 3-D beam finite element with warping displacements and its modeling capabilities
}

\author{
Kyungho Yoon, Youngyu Lee and Phill-Seung Lee* \\ Division of Ocean Systems Engineering, Korea Advanced Institute of Science and Technology, \\ Yuseong-gu, Daejeon 305-701, Korea
}

(Received February 9, 2012, Revised June 22, 2012, Accepted July 12, 2012)

\begin{abstract}
In this paper, we propose a continuum mechanics based 3-D beam finite element with crosssectional discretization allowing for warping displacements. The beam element is directly derived from the assemblage of 3-D solid elements, and this approach results in inherently advanced modeling capabilities of the beam element. In the beam formulation, warping is fully coupled with bending, shearing, and stretching. Consequently, the proposed beam elements can consider free and constrained warping conditions, eccentricities, curved geometries, varying sections, as well as arbitrary cross-sections (including thin/thick-walled, open/closed, and single/multi-cell cross-sections). We then study the modeling and predictive capabilities of the beam elements in twisting beam problems according to geometries, boundary conditions, and cross-sectional meshes. The results are compared with reference solutions obtained by analytical methods and solid and shell finite element models. Excellent modeling capabilities and solution accuracy of the proposed beam element are observed.
\end{abstract}

Keywords: beams; finite elements; torsion; twisting; warping; arbitrary cross-sections

\section{Introduction}

Beams are abundantly used in such areas as the construction of bridges, offshore jackets, roofs, and high-rise buildings (Timoshenko 1970). In practice, the finite element method has been predominantly used for the analysis of beam structures (Bathe 1996). The analysis methods for straight and prismatic beams have been well developed for a long time. However, for analyses of general beams with curved geometries, varying sections, and arbitrary cross-sectional shapes, use of more complicated models incorporating solid and shell finite elements is required. Wind turbine blades and airplane wings are good practical examples.

Beams can be modeled by 3-D solid and shell finite elements regardless of beam geometries. However, in practice, many structures have a large number of beam members, and the use of solid and shell finite elements to model the beams is not effective due to the large modeling effort and the computational time required.

Shell elements that are derived from 3-D solid elements are called continuum mechanics based shell finite elements, and they have been very successfully and dominantly used for the analysis of

*Corresponding author, Associate Professor, E-mail: phillseung@kaist.edu 
general shell structures. For examples, see Refs. (Dvorkin and Bathe 1984, Chapelle and Bathe 2003, Lee and Bathe 2004). Therefore, we can use a similar approach to formulate beam finite elements from 3-D solid finite elements. The well-known isoparametric beam finite elements are derived from a single 3-D solid element but, in general, only rectangular cross-sections can be considered (Bathe 1996). Lee and McClure developed isoparametric beam finite elements with angle cross-sections by embedding the cross-sections within rectangular cross-sectional domains (Lee and McClure 2006). Lee and Noh extended the approach to model tubular and wide flange cross-section beams (Lee and Noh 2010).

In general, when a non-circular cross-section beam is subjected to twisting, the beam crosssections do not remain plane during the deformations, and out-of-plane displacements occur (Timoshenko 1970). The effect is called warping. The behavior of warping is coupled with bending, shearing, and stretching under various loading conditions. The twisting and the coupling behaviors cannot be accurately predicted without considering warping. The inclusion of the warping effect in the beam element formulation is very important. To consider the fully coupled warping effect, the warping displacements can be included in the beam element formulation as additional displacement fields with corresponding degrees of freedom, see Refs. (Bathe and Chaudhary 1982, Dutta and White 1992).

While the warping effect for thin-walled beams has been extensively studied for a long time, see Refs. (Vlasov 1961, Gjelsvik 1981, Prokić 1996, Gonçalves et al. 2010), and therein, relatively little effort has been expended for modeling the warping of beams with arbitrary cross-sections. To effectively consider both arbitrary cross-sections and warping effects, a concept similar to the continuum mechanics based approach has been used in Refs. (Lee and Kim 1987, Živkovic et al. 2001), although the term "continuum mechanics based" was not used in those papers. Lee and Kim introduced a beam finite element with cross-sectional discretization, where warping degrees of freedom are defined at all cross-sectional nodes as unknowns (Lee and Kim 1987). The beam element is general but there were many additional degrees of freedom used for warping displacements at each beam node. ivkoviæ et al. developed a more general beam finite element with deformable cross-sections (Živkovic et al. 2001). It can be considered a superelement and is obtained by assembling various solid, beam, and shell finite elements. Therefore, solid, beam, and shell finite elements are required in advance to implement the beam superelement.

In the present study, we develop a general and efficient 3-D beam finite element with crosssectional discretizations that allows for warping displacements and study the twisting behavior of the beam element under various modeling conditions. We refer to this beam finite element as "a continuum mechanics based beam finite element." The beam element is derived from the assemblage of 3-D solid elements but is not a superelement. The proposed beam element has great modeling capabilities to deal with complicated beam geometries.

The novel features of the proposed beam element that originate from the inherent generality of the continuum mechanics based approach are as follows:

- The formulation is simple and straightforward.

- The formulation can handle all complicated 3-D geometries including curved geometries, varying cross-sections, and arbitrary cross-sectional shapes (including thin/thick-walled and open/ closed cross-sections).

- Warping effects fully coupled with bending, shearing, and stretching are automatically included.

- Seven degrees of freedom (only one additional degree of freedom for warping) are used at each beam node to ensure inter-elemental continuity of warping displacements. 
- The pre-calculation of cross-sectional properties (area, second moment of area, etc.) is not required because the beam formulation is based on continuum mechanics.

- Analyses of short, long, and deep beams are available, and eccentricities of loadings and displacements on beam cross-sections are naturally considered.

- The basic formulation can be easily extended to general nonlinear analyses that consider geometrical and material nonlinearities.

In the following sections, we first present a derivation of the continuum mechanics based beam finite element with warping displacements. We then show how to properly construct warping displacement fields. In numerical studies, we focus on presenting the twisting behaviors of the beam finite element considering various geometries, loading and boundary conditions, and cross-sectional meshes used. The results of various numerical tests are given including comparisons with analytical and solid and shell model reference solutions (Dvorkin and Bathe 1984, Lee and Bathe 2004, Lee and Bathe 2005).

\section{Finite element discretization}

In this section, we present the formulation of the continuum mechanics based beam finite element. The beam formulation is derived from the assemblage of solid finite elements.

\subsection{Interpolation of geometry}

An arbitrary geometry of a beam can be modeled by 3-D solid finite elements aligned in the beam length direction as shown in Fig. 1. Here, all the nodes of the solid elements are positioned on several cross-sectional planes of the beams (for example, three cross-sectional planes in Fig. 1). The geometry interpolation of the $l$-node solid element $m$ is given by

$$
\vec{x}^{(m)}=\sum_{i=1}^{l} h_{i}(r, s, t) \vec{x}_{i}^{(m)}
$$

where $\vec{x}^{(m)}$ is the material position vector of the solid element $m$ in the global Cartesian coordinate system, $h_{i}(r, s, t)$ are the 3 -D interpolation polynomials for the usual isoparametric procedure (that is, shape functions) and $\vec{x}_{i}^{(m)}$ is the $i$ th nodal position vector of the solid element $m$.

Since the nodes of the solid element are placed on the cross-sectional planes, the 3-D interpolation in Eq. (1) can be replaced by the multiplication of 1-D and 2-D shape functions,

$$
\vec{x}^{(m)}=\sum_{k=1}^{q} h_{k}(r) \sum_{j=1}^{p} h_{j}(s, t) \vec{x}_{k}^{j(m)}
$$

in which $q$ is the number of the cross-sectional planes, $p$ is the number of the nodes of the solid element $m$ positioned at each cross-sectional plane (for example, $q=3$ and $p=9, l=p \times q=27$ for the 27-node solid element $m$ shaded in Fig. $1, h_{k}(r)$ and $h_{j}(s, t)$ are the 1-D and 2-D interpolation polynomials for the usual isoparametric procedure, respectively, and $\vec{x}_{k}^{j(m)}$ are the $j$ th nodal position vector of the solid element $m$ on cross-sectional plane $k$, see Fig. 1 . Here, we call $\vec{x}_{k}^{j(m)}$ the position vector of the $j$ th cross-sectional node on cross-sectional plane $k$ corresponding to 


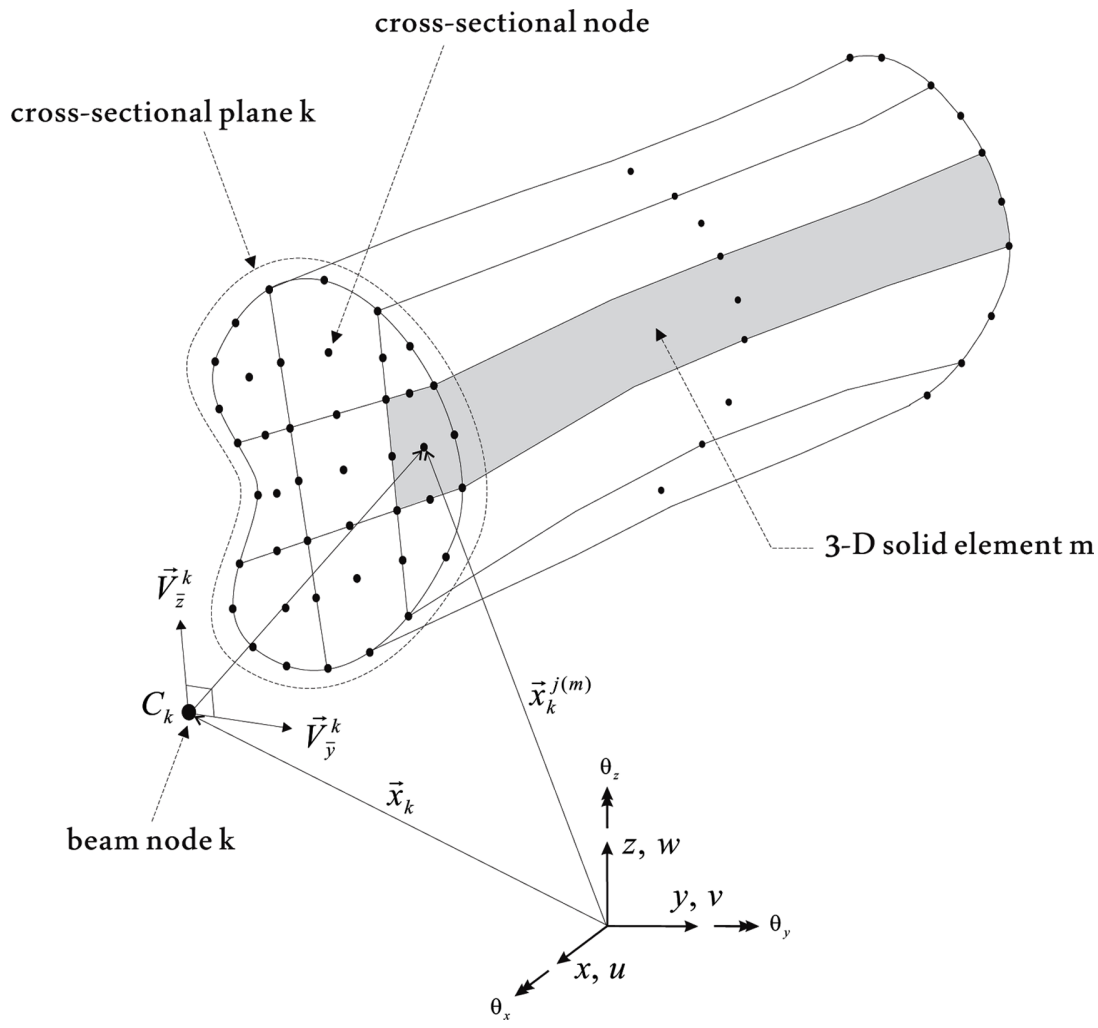

Fig. 1 The concept of the continuum mechanics based beam finite element with sectional discretization. In this figure, the beam is modeled by nine 3-D solid elements and the model has 3 cross-sectional planes along the beam length. The model in the figure becomes a 3-node beam finite element with cross-sectional discretization

the solid element $m$. In general, the order of the 2-D interpolation function does not need to be the same as the order of the 1-D interpolation function.

The basic kinematic assumption of classical beam theories is that plane cross-sections originally normal to the mid-line of the beam remain plane and undistorted during deformations but not necessarily normal to the mid-line of the deformed beam (Timoshenko 1970). This assumption of the Timoshenko beam theory can be enforced at all the cross-sectional nodes (Živkovic et al. 2001)

$$
\vec{x}_{k}^{j(m)}=\vec{x}_{k}+\bar{y}_{k}^{j(m)} \vec{V}_{\bar{y}}^{k}+\bar{z}_{k}^{j(m)} \vec{V}_{\bar{z}}^{k}
$$

where the unit vectors $\vec{V}_{\bar{y}}^{k}$ and $\vec{V}_{\bar{z}}^{k}$ are the director vectors placed on cross-sectional plane $k$, the two vectors and the position $\vec{x}_{k}$ of origin at point $C_{k}$ define the cross-sectional Cartesian coordinate system, and $\bar{y}_{k}^{j(m)}$ and $\bar{z}_{k}^{j(m)}$ represent the material position of the $j$ th cross-sectional node of the solid element $m_{k}$ in the cross-sectional Cartesian coordinate system on cross-sectional plane $k$. Note that $\vec{V}_{\bar{y}}^{k}$ and $\vec{V}_{\bar{z}}^{k}$ are normal to each other and determine the direction of cross-sectional plane $k$. The vector relation in Eq. (3) is graphically represented in Fig. 1.

The use of Eq. (3) in Eq. (2) results in the geometry interpolation of the $q$-node continuum mechanics based beam finite element corresponding to the solid element $m$ 


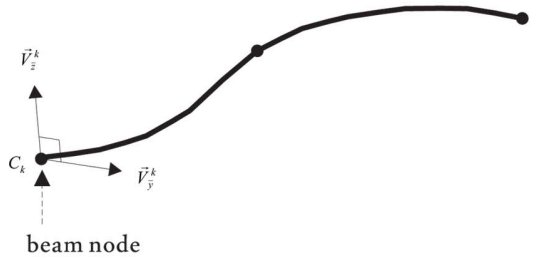

(a)

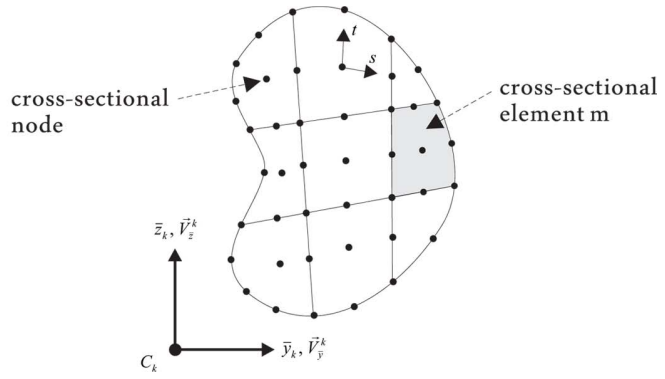

(b)

Fig. 2 Continuum mechanics beam finite element with cross-sectional mesh. (a) 3-node beam finite element, (b) cross-sectional mesh at beam node $k$

$$
\vec{x}^{(m)}=\sum_{k=1}^{q} h_{k}(r) \vec{x}_{k}+\sum_{k=1}^{q} h_{k}(r) \bar{y}_{k}^{(m)} \vec{V}_{\bar{y}}^{k}+\sum_{k=1}^{q} h_{k}(r) \bar{z}_{k}^{(m)} \vec{V}_{\bar{z}}^{k}
$$

with

$$
\bar{y}_{k}^{(m)}=\sum_{j=1}^{p} h_{j}(s, t) \bar{y}_{k}^{j(m)}, \quad \bar{z}_{k}^{(m)}=\sum_{j=1}^{p} h_{j}(s, t) \bar{z}_{k}^{j(m)}
$$

where $\bar{y}_{k}^{(m)}$ and $\bar{z}_{k}^{(m)}$ denote the material position of the solid element $m$ in the cross-sectional Cartesian coordinate system on cross-sectional plane $k$. Eq. (5) indicates that the material position on the cross-sectional plane is interpolated by cross-sectional nodes. It is important to know that Eq. (4) is the geometry interpolation of the solid element $m$ aligned in the beam length direction in which the kinematic assumption of the Timoshenko beam theory is enforced.

Then, the point $C_{k}$ corresponds to the $k$ th beam node. The beam node at point $C_{k}$ can be arbitrarily positioned on cross-sectional plane $k$ defined by the two director vectors $\vec{V}_{\bar{y}}^{k}$ and $\vec{V}_{\bar{z}}^{k}$ in Fig. 1. The longitudinal reference line that is used to define the geometry of the beam is created by connecting the beam nodes. In this paper, it is important to know the difference between beam nodes and cross-sectional nodes, see Figs. 1 and 2.

As mentioned, the geometry interpolation of the beam element in Eq. (4) corresponds to the solid element $m$. The simple assemblage of the interpolation functions corresponding to all the solid elements aligned along the beam length direction represents the geometry interpolation of the whole beam element. As a result, the beam finite element shown in Fig. 2(a) can have a cross-sectional discretization in Fig. 2(b) at each beam node. The size and shape of the cross-sections can arbitrarily vary but the cross-sectional mesh pattern should be the same to maintain the continuity of the geometry on all the cross-sectional planes.

\subsection{Interpolation of displacements}

From the interpolation of geometry in Eq. (4), the interpolation of displacements corresponding to the solid element $m$ is derived as in (Bathe 1996)

$$
\vec{u}^{(m)}=\sum_{k=1}^{q} h_{k}(r) \vec{u}_{k}+\sum_{k=1}^{q} h_{k}(r) \bar{y}_{k}^{(m)}\left\{\vec{\theta}_{k} \times \vec{V}_{\bar{y}}^{k}\right\}+\sum_{k=1}^{q} h_{k}(r) \bar{z}_{k}^{(m)}\left\{\vec{\theta}_{k} \times \vec{V}_{\bar{z}}^{k}\right\}
$$


in which $\vec{u}_{k}$ and $\vec{\theta}_{k}$ are the displacement and rotation vectors, respectively, in the global Cartesian coordinate system at beam node $k$

$$
\vec{u}_{k}=\left\{\begin{array}{c}
u^{k} \\
v^{k} \\
w^{k}
\end{array}\right\} \quad \text { and } \quad \vec{\theta}_{k}=\left\{\begin{array}{c}
\theta_{x}^{k} \\
\theta_{y}^{k} \\
\theta_{z}^{k}
\end{array}\right\}
$$

Eq. (6) indicates that the displacement fields of all the solid elements that compose the whole beam is determined by the three translations and three rotations (six degrees of freedom) at each beam node because the nodes of the solid elements are placed on the cross-sectional planes and the kinematic assumption of the Timoshenko beam theory is enforced. Therefore, the assemblage of the solid elements can act like a single beam element and the beam element can have the crosssectional discretization.

The basic displacement field in Eq. (6) can be enriched by adding various displacement patterns and then the generalized interpolation of displacements is obtained

$$
\vec{u}_{g}^{(m)}=\vec{u}^{(m)}+\vec{u}_{a}^{(m)}
$$

in which $\vec{u}_{a}^{(m)}$ is the additional interpolation of displacements, which could include warping displacements, displacements for cross-sectional distortions and so on.

\subsection{Interpolation of warping displacements}

In order to consider the warping displacements, we use the generalized interpolation of displacements

with

$$
\begin{gathered}
\vec{u}_{g}^{(m)}=\vec{u}^{(m)}+\vec{u}_{w}^{(m)} \\
\vec{u}_{w}^{(m)}=\sum_{k=1}^{q} h_{k}(r) f_{k}^{(m)}(s, t) \vec{V}_{r}^{k}
\end{gathered}
$$

where $\vec{V}_{r}^{k}$ is the director vector normal to cross-sectional plane $k$ and is defined by the director vectors $\vec{V}_{\bar{y}}^{k}$ and $\vec{V}_{\bar{z}}^{k}$

$$
\vec{V}_{r}^{k}=\frac{\vec{V}_{\bar{y}}^{k} \times \vec{V}_{\bar{z}}^{k}}{\left|\vec{V}_{\bar{y}}^{k} \times \vec{V}_{\bar{z}}^{k}\right|} \quad \text { or } \quad \vec{V}_{r}^{k}=\frac{\vec{V}_{\bar{y}}^{k} \times \vec{V}_{\bar{z}}^{k}}{\left|\vec{V}_{\bar{y}}^{k} \times \vec{V}_{\bar{z}}^{k}\right|}
$$

The sign of the directions in Eq. (11) should be carefully chosen to enforce the continuity of the longitudinal displacements at beam nodes shared by beam elements. Figs. 3(a) and (b) show the correct and wrong warping directions, respectively.

Note that the numerical solutions depend on the directions of the warping displacements chosen. When varying cross-sections are considered, the warping directions we use correspond to Fig. 3(d). Figs. 3(c) and (e) show alternative choices of the warping directions, which are defined by the longitudinal direction of the beam elements, but the directions in Figs. 3(a) and (d) give better numerical results, in particular, when few beam elements are used. Some numerical results that show the effect of the warping direction will be presented in numerical studies. 
(a)

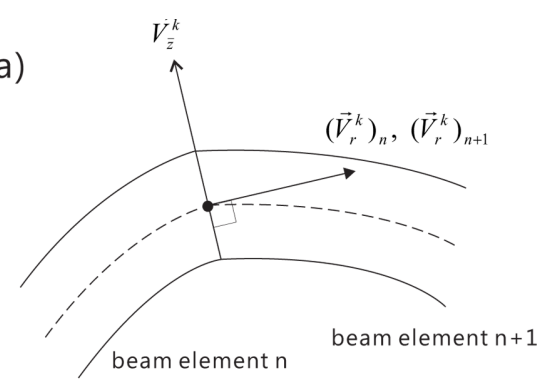

(c)

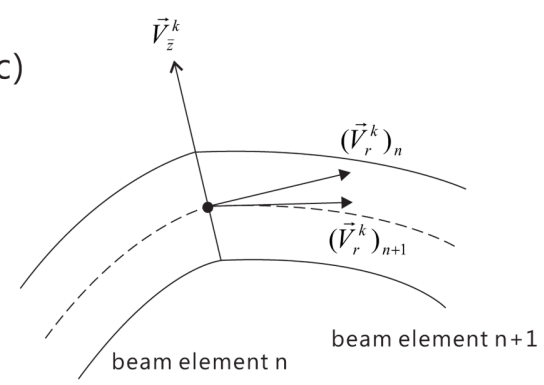

(b)

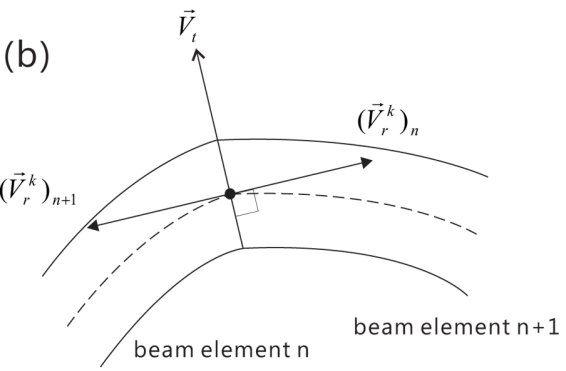

(d)

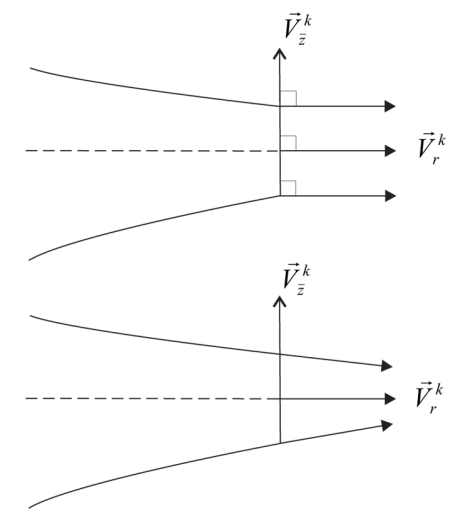

Fig. 3 Directions of warping displacements. (a) and (b) correct and wrong warping directions at a sharing node, (c) warping direction same to the longitudinal direction of beam elements, (d) and (e) uniform and varying warping directions at varying beam cross-sections

Based on the cross-sectional discretization at beam node $k$ in Fig. 2(b), we introduce the interpolation of warping displacements. The natural choice is to use

$$
f_{k}^{(m)}(s, t)=\sum_{j=1}^{p} h_{j}(s, t) f_{k}^{j(m)}
$$

where $f_{k}^{j(m)}$ are warping degrees of freedom at beam node $k$ corresponding to the solid element $m$. In this case, the total number of degrees of freedom for warping at each beam node is the same as the number of cross-sectional nodes on each cross-sectional plane. Therefore, Eq. (12) requires many additional warping degrees of freedom at beam nodes depending on the cross-sectional meshes used and the resulting element is similar to the beam element in (Lee and Kim 1987).

In order to use only one additional degree of freedom for warping displacements at each beam node, we then use

$$
f_{k}^{(m)}(s, t)=\sum_{j=1}^{p} h_{j}(s, t) f_{k}^{j(m)} \alpha_{k}
$$

in which $\alpha_{k}$ is the warping degree of freedom at beam node $k$ and $f_{k}^{j(m)}$ is the pre-calculated warping values on cross-sectional plane $k$ by solving St. Venant equations. Then, the continuum mechanics based beam finite element with cross-sectional discretization that allows for the warping effect has only seven degrees of freedom at each beam node. Through the warping degree of 
freedom $\alpha_{k}$ in Eq. (13), the inter-elemental continuity of the warping displacements is ensured since adjacent beam elements connect to the same beam node. Without considering the inter-elemental continuity, non-uniform warping behaviors along beam length due to non-uniform torsion or constrained warping conditions cannot be properly predicted.

Note that the interpolation of warping displacements in Eq. (12) does not require pre-calculation, that is, the warping is automatically considered in the beam formulation. However, a special treatment is required to remove redundant rigid body modes that occur due to the warping degrees of freedoms $f_{k}^{j(m)}$ (Lee and Kim 1987).

\subsection{Calculation of warping values}

Here we present the procedure for calculating the warping values $f_{k}^{j(m)}$ in Eq. (13). The warping values are numerically calculated by solving St. Venant equations in the 2-D cross-sectional domain $\Omega_{k}$ with its boundary $\partial \Omega_{k}$ on cross-sectional plane $k$, see Fig. 4

$$
\begin{gathered}
\frac{\partial^{2} \bar{f}_{k}}{\partial \bar{y}_{k}^{2}}+\frac{\partial^{2} \bar{f}_{k}}{\partial \bar{z}_{k}^{2}}=0 \quad \text { in } \quad \Omega_{k} \\
n_{\bar{y}}^{k} \frac{\partial \bar{f}_{k}}{\partial \bar{y}_{k}}+n_{\bar{z}}^{k} \frac{\partial \bar{f}_{k}}{\partial \bar{z}_{k}}=n_{\bar{y}}^{k} \bar{z}_{k}-n_{\bar{z}}^{k} \bar{y}_{k} \quad \text { in } \quad \partial \Omega_{k}
\end{gathered}
$$

in which $\bar{f}_{k}$ is the warping function defined in the cross-sectional Cartesian coordinate system with the point of origin $C_{k}$, and $n_{\bar{y}}^{k}$ and $n_{\bar{z}}^{k}$ are the components of the vector normal to the crosssectional boundary $\partial \Omega_{k}$. Note that, in general, the position of origin $C_{k}$ does not coincide with the center of twist of the cross-sectional plane.

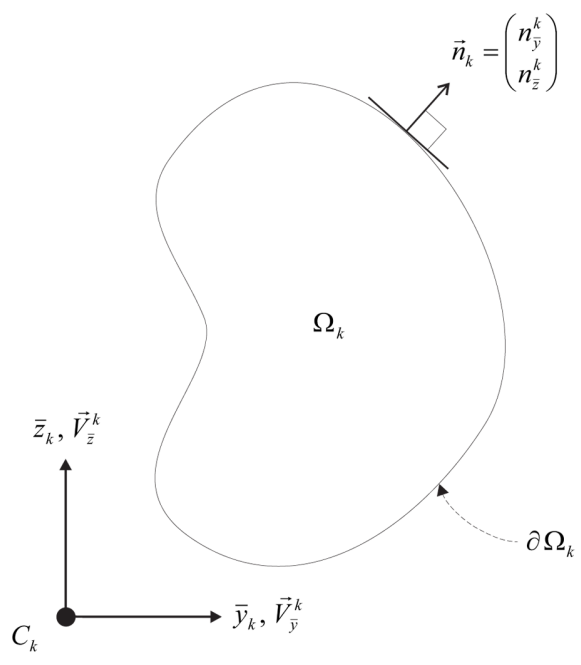

(a)

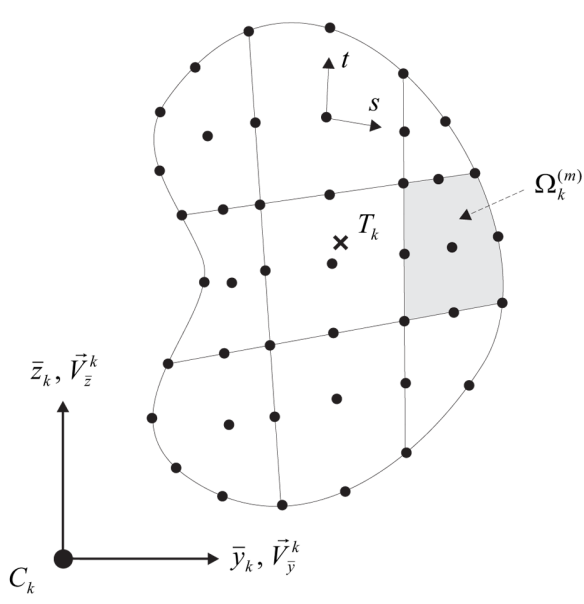

(b)

Fig. 4 A cross-section to solve St. Venant equations. (a) cross-sectional Cartesian coordinate system (point of origin $C_{k}$ ) and a vector normal to cross-sectional boundary, (b) cross-sectional mesh and the center of twist $T_{k}$ 
St. Venant equations can be easily solved by the standard finite element formulation

$$
\int_{\Omega_{k}}\left(\frac{\partial \bar{f}_{k}}{\partial \bar{y}_{k}} \frac{\partial \delta \bar{f}_{k}}{\partial \bar{y}_{k}}+\frac{\partial \bar{f}_{k}}{\partial \bar{z}_{k}} \frac{\partial \delta \bar{f}_{k}}{\partial \bar{z}_{k}}\right) d a=\int_{\partial \Omega_{k}}\left(n_{\bar{y}}^{k} \bar{z}_{k}-n_{\bar{z}}^{k} \bar{y}_{k}\right) \overline{\delta f} k d s
$$

where $\delta \bar{f}_{k}$ is the variation of the warping function discretized by the same mesh as shown in Fig. 2(b) and the interpolation of warping in the cross-sectional element $m$ (corresponding to the solid element $m$ ) is the same as in Eqs. (5), (12) and (13)

$$
\bar{f}_{k}^{(m)}=\sum_{j=1}^{p} h_{j}(s, t) \bar{f}_{k}^{j(m)}
$$

where $\bar{f}_{k}^{j(m)}$ are the values of the warping function at cross-sectional nodes. Note that when we solve Eq. (16), one warping degree of freedom that is arbitrarily chosen should be prescribed to avoid the singularity of the system matrix obtained by Eq. (16).

If the origin of the cross-sectional Cartesian coordinate system $C_{k}$ is moved, the boundary condition term in the right hand side of Eqs. (15) and (16) is changed. As a result, the solution of St. Venant equations (warping function $f_{k}^{(m)}$ ) depends on where we position the origin of the crosssectional Cartesian coordinate system.

The proper warping function $f_{k}^{(m)}$ is the solution calculated when the center of twist coincides with the origin of the cross-sectional Cartesian coordinate system because the beam cross-sections subjected to torsion rotate about the center of twist with the resulting warping. Therefore, the proper warping function $\left(f_{k}^{(m)}\right)$ corresponding to the center of twist should be transformed from the warping function $\left(\bar{f}_{k}^{(m)}\right)$ corresponding to the origin $C_{k}$

$$
\begin{gathered}
f_{k}^{(m)}=M\left(\bar{f}_{k}^{(m)}\right)+\bar{y}_{k}^{T} M\left(\bar{z}_{k}^{(m)}\right)-\bar{z}_{k}^{T} M\left(\bar{y}_{k}^{(m)}\right) \\
M\left(g^{(m)}\right)=g^{(m)}-\frac{1}{A} \sum_{m=1}^{d} \int_{\Omega_{k}^{(m)}} g^{(m)} d a
\end{gathered}
$$

with

where $\bar{y}_{k}^{T}$ and $\bar{z}_{k}^{T}$ represent the position of the center of twist $T_{k}$ on cross-sectional plane $k, \Omega_{k}^{(m)}$ is the domain of the cross-sectional element $m, d$ is the number of cross-sectional elements and $A$ is the cross-sectional area $A=\sum_{m=1}^{d} \int_{\Omega_{k}^{(m)}} d a$. This is the key step to reach an effective element formulation.

From the solution of the warping function $\left(\bar{f}_{k}^{(m)}\right)$ corresponding to the origin $C_{k}$, the position of the center of twist in Eq. (18) can be calculated (Gruttmann et al. 1999) by

$$
\begin{aligned}
& \bar{y}_{k}^{T}=\frac{I\left(\bar{f}_{k}^{(m)}, \bar{y}_{k}^{(m)}\right) I\left(\bar{y}_{k}^{(m)}, \bar{z}_{k}^{(m)}\right)-I\left(\bar{f}_{k}^{(m)}, \bar{z}_{k}^{(m)}\right) I\left(\bar{y}_{k}^{(m)}, \bar{y}_{k}^{(m)}\right)}{I\left(\bar{y}_{k}^{(m)}, \bar{y}_{k}^{(m)}\right) I\left(\bar{z}_{k}^{(m)}, \bar{z}_{k}^{(m)}\right)-I^{2}\left(\bar{y}_{k}^{(m)}, \bar{z}_{k}^{(m)}\right)} \\
& \bar{z}_{k}^{T}=\frac{I\left(\bar{f}_{k}^{(m)}, \bar{y}_{k}^{(m)}\right) I\left(\bar{z}_{k}^{(m)}, \bar{z}_{k}^{(m)}\right)-I\left(\bar{f}_{k}^{(m)}, \bar{z}_{k}^{(m)}\right) I\left(\bar{y}_{k}^{(m)}, \bar{z}_{k}^{(m)}\right)}{I\left(\bar{y}_{k}^{(m)}, \bar{y}_{k}^{(m)}\right) I\left(\bar{z}_{k}^{(m)}, \bar{z}_{k}^{(m)}\right)-I^{2}\left(\bar{y}_{k}^{(m)}, \bar{z}_{k}^{(m)}\right)}
\end{aligned}
$$

in which an integration operator $I$ is defined by 


$$
I\left(g_{1}^{(m)}, g_{2}^{(m)}\right)=\sum_{m=1}^{d} \int_{\Omega_{k}^{(m)}}\left[M\left(g_{1}^{(m)}\right) M\left(g_{2}^{(m)}\right)\right] d a
$$

All the integrations in Eqs. (18), (19) and (20) are numerically calculated.

It is important to note that, although the calculated center of twist is the approximated one for the actual beam cross-section, it is the exact center of twist for the discretized beam cross-section; that is, when torsion is applied at the center of twist in the discretized beam cross-section, no transverse displacements occur.

\subsection{Numerical integration}

With the interpolations of the geometry and the displacements given, the procedure to construct the strain-displacement matrix, the stiffness matrix and the load vector is standard as in (Bathe 1996).

In a beam cross-section ( $s-t$ plane), the standard numerical integration procedure is used. For example, the $2 \times 2$ Gauss integration is used for 4-node cross-sectional elements and the $3 \times 3$ Gauss integration is used for 9-node cross-sectional elements.

When the width and height of a beam cross-section are small compared to the beam length, the beam finite element locks, that is, the element is too stiff in bending. However, the locking can be easily removed using a mixed formulation that is effectively implemented by using reduced integration corresponding to the $r$-direction (Bathe 1996, Chapelle and Bathe 2003). We use this procedure in our implementation.

\section{Numerical studies}

To verify the proposed formulation and to study the twisting behavior of the beam element depending on geometries, boundary conditions and cross-sectional meshes, we perform various numerical analyses using our beam elements. The results are compared with available analytical solutions and solutions of equivalent finite element models. Young's modulus $E=2.0 \times 10^{11} \mathrm{~N} / \mathrm{m}^{2}$ and Poisson's ratio $v=0$ are used for all the beam problems considered in this section ${ }^{\mathrm{a}}$.

\subsection{Rectangular cross-section beam problems}

A straight cantilever beam of $L=20 \mathrm{~m}$ with a rectangular cross-section is subjected to torsional moment $M_{x}=1.0 \mathrm{~N} \cdot \mathrm{m}$ at the center of twist at the free tip $(x=L)$ as shown in Fig. 5(a). Then, uniform torsion occurs on all the beam cross-sections. Various aspect ratios of width to height $(a / b)$ are considered for the rectangular cross-section, see Fig. 6. Warping is free at both ends and along the beam length. The boundary condition is given as

$$
u=v=w=\theta_{x}=\theta_{y}=\theta_{z}=0 \quad \text { at } x=0
$$

${ }^{a}$ In order to avoid difficulties in specifying the boundary conditions of equivalent shell and solid finite element models, zero Poisson's ratio is used. 


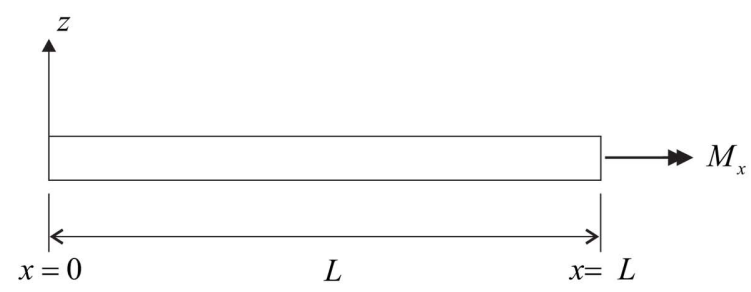

(a)

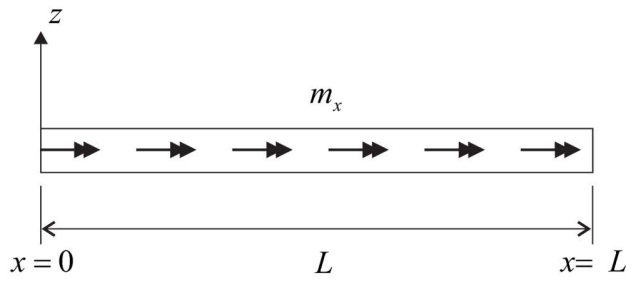

(b)

Fig. 5 Straight cantilever beam problems (a) uniform torsion problem (a straight beam subjected to torsional moment at the free tip) (b) non-uniform torsion problem (a straight beam subjected to uniformly distributed torsional moment)
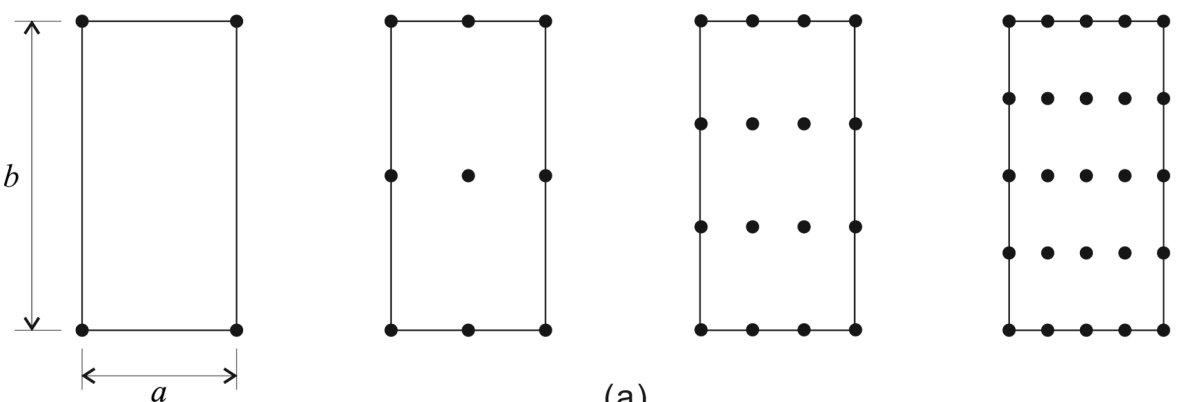

(a)
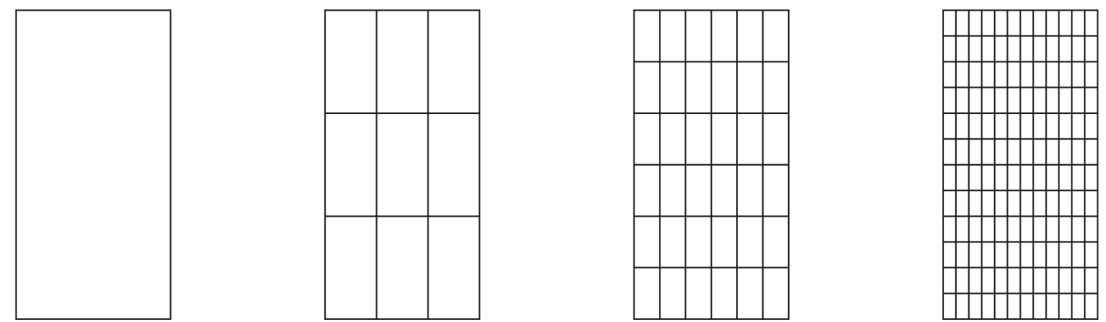

(b)
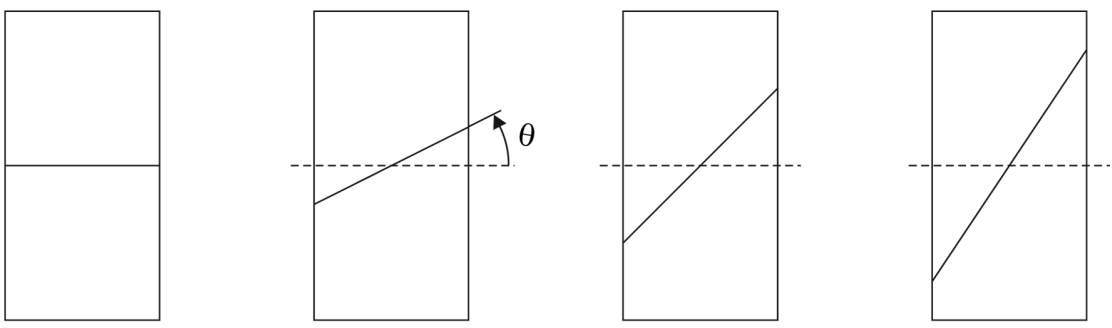

(c)

Fig. 6 Various cross-sectional discretizations with different interpolation functions and meshes. (a) single element meshes (1st, 2nd, 3rd and 4th order interpolations), (b) $1 \times 1,3 \times 3,6 \times 6$ and $12 \times 12$ linear meshes, (c) distorted meshes $(\tan \theta=0, b / 4 a, 2 b / 4 a$ and $3 b / 4 a)$ of 16-node cross-sectional elements 


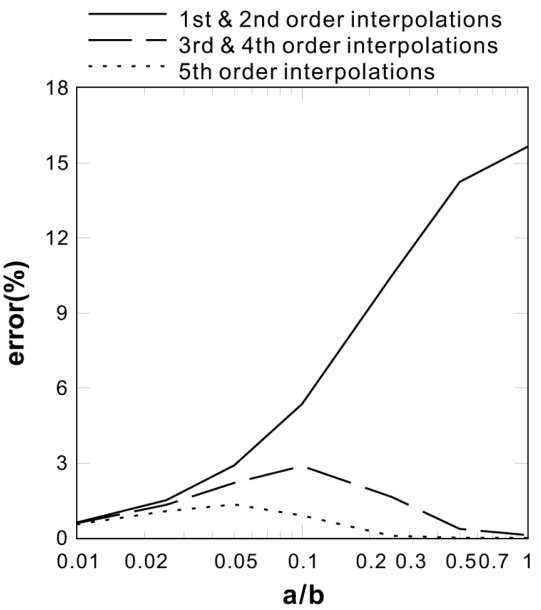

(a)

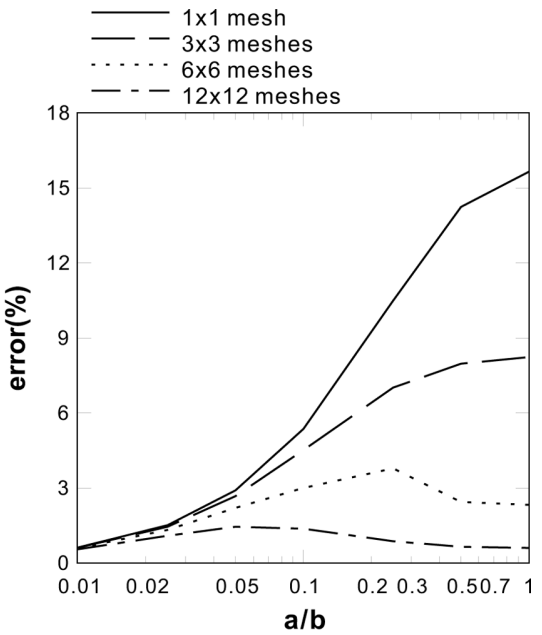

(b)

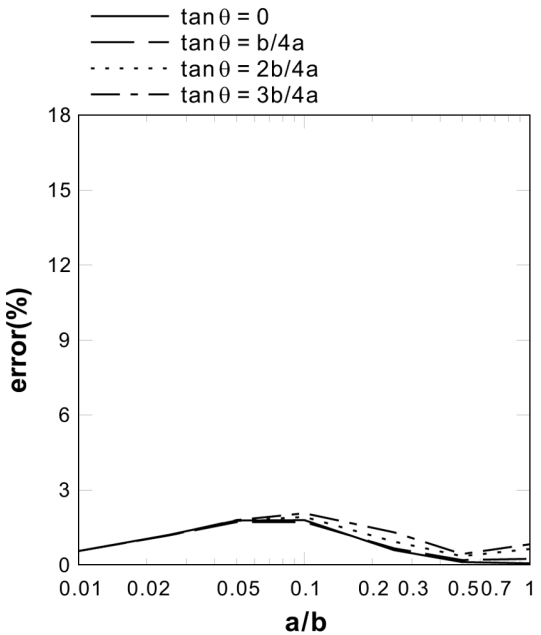

(c)

Fig. 7 Relative errors in angle of twist at the loaded tip for various aspect ratios $(a / b)$; (a) corresponding to the interpolation orders in Fig. 6(a); (b) corresponding to the meshes of linear cross-sectional elements in Fig. 6(b); (c) corresponding to the distorted cross-sectional meshes in Fig. 6(c)

We model the problem with the proposed single 2-node beam finite element, and various crosssectional discretizations shown in Fig. 6 are tested. It is very important to note that in uniform torsion problems of prismatic beams with free warping, the same solutions are obtained regardless of the number of beam elements and the order of beam elements used. The angles of twist in these numerical calculations mainly depend on the cross-sectional mesh used. In Fig. 7, we plot the errors in the angle of twist at the loaded tip using the analytical solutions in Ref. (Timoshenko 1970).

Fig. 7(a) shows the errors according to the order of finite elements used to model the crosssection corresponding to the meshes in Fig. 6(a). It is very interesting to note that the 1 st and 2 nd order interpolations (4-node and 9-node cross-sectional elements) and the 3rd and 4th order 
interpolations (16-node and 25-node cross-sectional elements) give the identical results, respectively. It can be expected that the warping functions of rectangular cross-sections have the characteristics of odd functions. When a single 16-node cross-sectional element is used, the error is less than $3 \%$ in the whole range of the aspect ratio $a / b$. The solutions are also very accurate in two extreme cases: the square cross-section $(a / b=1)$ and the thin cross-section $(a / b \ll 1)$. A more accurate solution by a single 36-node cross-sectional element (5th order interpolation) is also presented.

Fig. 7(b) shows the error in the angle of twist when $1 \times 1,3 \times 3,6 \times 6$ and $12 \times 12$ meshes of 4 -node cross-sectional elements in Fig. 6(b) are used. The linear element gives good accuracy for the thin cross-section case. While, as the mesh is refined, the error decreases in the whole range of $a / b$, the solutions of the $12 \times 12$ mesh of the linear elements are not better than those of a single 36-node cross-sectional element.

Through the two tests, we can conclude that the use of higher order cross-sectional elements is more effective than use of many lower order cross-sectional elements. The use of the 16-node elements for cross-sectional discretizations gives reasonable accuracy for general engineering purposes.

In general, thin-walled beams are composed of several thin plates that have a ratio $a / b<0.1$, and the graphs in Fig. 7(a) show that the 4-node cross-sectional element gives 5\% and 9\% errors for $a / b=0.1$ and $a / b=0.2$, respectively. The use of one 4-node cross-sectional element for each plate section of thin-walled beams gives solutions accurate enough for engineering practices.

Additionally, Fig. 7(c) reveals that the effect of mesh distortion on error in the angle of twist is not considerable when 16-node cross-sectional elements are used.

\subsection{Tubular and angle cross-section beam problems}

Considering the tubular and angle cross-sections in Fig. 8, we analyze the straight cantilever beam problem of length $L=20$ in Fig. 5. Torsional moment $M_{x}=1.0 \mathrm{~N} \cdot \mathrm{m}$ is applied at the center of twist at $x=L$ for the tubular cross-section problems as in Fig. 5(a) and uniformly distributed torsional moment $m_{x}=1.0 \mathrm{~N} \cdot \mathrm{m} / \mathrm{m}$ is applied at the center of twist along the beam length for the angle cross-section problems as in Fig. 5(b). For both problems, the warping of the beam is constrained at the fixed end

$$
u=v=w=\theta_{x}=\theta_{y}=\theta_{z}=\alpha=0 \quad \text { at } x=0
$$

As shown in Fig. 8, for both cross-sections, the cross-sectional dimensions $a$ and $b$ are fixed and three different thicknesses $(t / a=0.1,0.5$ and 0.999$)$ are considered. As the ratio $t / a$ goes to 1.0 , the cross-sections get closer to a rectangular cross-section. Each tubular cross-section is modeled by eight cross-sectional elements and each angle cross-section is modeled by three cross-sectional elements, see Fig. 8.

Reference solutions are calculated by the 27-node solid element models in Fig. 9. Note that the solid element solutions depend on how we apply the torsional moment on the solid element models. A special point in the modeling is that the torsional moments need to be imposed appropriately. We assign the loads described in Fig. 9, where

$$
l_{i} \cdot p_{i}=M_{i}=\frac{K_{i}}{K_{t}} M
$$




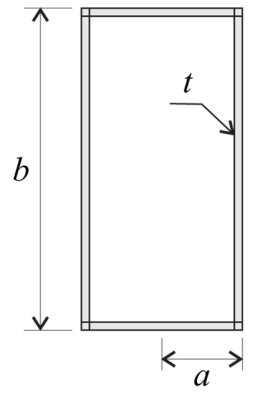

$t / a=0.1$

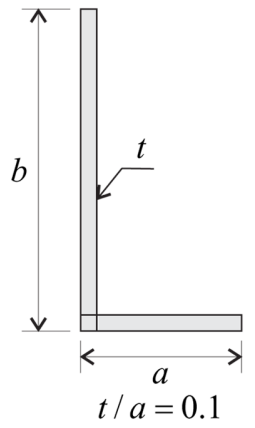

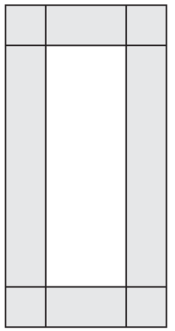

$t / a=0.5$

(a)

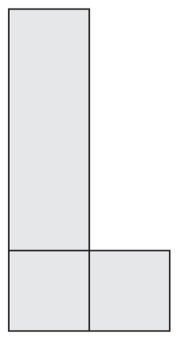

$t / a=0.5$

(b)

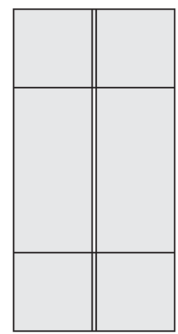

$t / a=0.999$

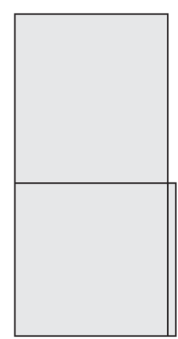

$t / a=0.999$

Fig. 8 Tubular and angle cross-sections with various thicknesses and cross-sectional meshes used. (a) Tubular cross-sections $(a=0.5, b=2)$, (b) Angle cross-sections $(a=1, b=2)$

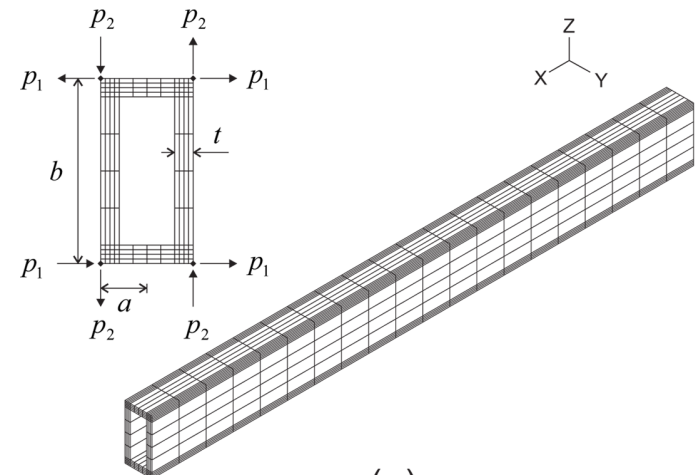

(a)

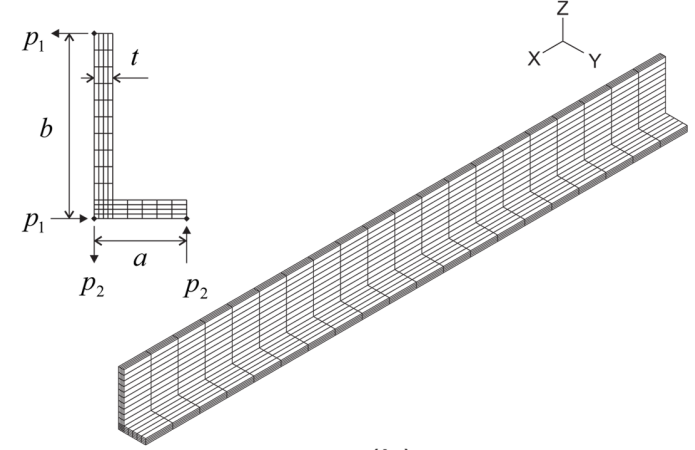

(b)

Fig. 9 Solid element models and meshes used. (a) Tubular cross-section beam with $128 \times 20$ meshes of 27 node solid elements $\left(p_{1}=0.125 \mathrm{~N}, p_{2}=0.25 \mathrm{~N}\right)$, (b) angle cross-section beam with $76 \times 20$ meshes of 27 -node solid elements $\left(p_{1}=0.0125 \mathrm{~N} / \mathrm{m}, p_{2}=0.025 \mathrm{~N} / \mathrm{m}\right)$

Here, $p_{i}$ is the point load exerting a couple-moment $M_{i}$ on plate section $i, l_{i}$ is the length of the plate section $i, K_{i}$ and $K_{t}$ are the torsional stiffnesses of the plate section $i$ and the complete crosssection, respectively, and $M$ is the total twisting moment applied. The tubular and angle crosssections in Fig. 9 consist of 4- and 2-plate sections, respectively. In order to approximate $K_{i}$ and $K_{t}$, 
we use the formulas available for thin-walled cross-section beams under free warping (Timoshenko 1970). The same rule is used for thick-walled cross-section cases.

Fig. 10 shows the method used to measure the angles of twist in the solid element models of the tubular and angle cross-section beam problems. The angles calculated from translational displacements at two sampling points as shown in Fig. 10 are averaged.

Tables 1 and 2 present the numerical results for the tubular cross-section beam problems under uniform torsion in the three different thickness cases. The use of 16-node cross-sectional elements gives good agreement with the reference solutions in all the thickness cases. Tables 3 and 4 show a similar investigation for the angle cross-section beam problems under non-uniform torsion. For both beam problems, two 2-node beam elements and one 3-node beam element give solutions accurate enough for engineering practice.
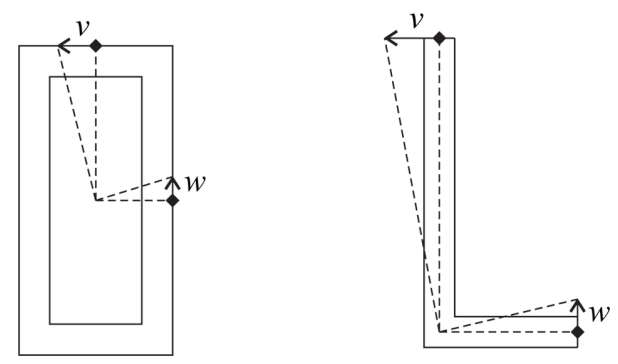

Fig. 10 Method to measure angles of twist in the solid element models of the tubular and angle cross-section beams

Table 1 Angles of twist at the loaded tips for tubular cross-section beam problems under uniform torsion with constrained warping. Eight 16-node cross-sectional elements are used and $N$ denotes the number of beam elements used

\begin{tabular}{|c|c|c|c|c|c|c|}
\hline \multirow{2}{*}{$N$} & \multicolumn{2}{|c|}{$t / a=0.1$} & \multicolumn{2}{|c|}{$t / a=0.5$} & \multicolumn{2}{|c|}{$t / a=0.999$} \\
\hline & 2-node beam & 3-node beam & 2-node beam & 3-node beam & 2-node beam & 3-node beam \\
\hline 1 & $1.6480 \mathrm{E}-09$ & $1.6466 \mathrm{E}-09$ & $5.1020 \mathrm{E}-10$ & $5.0960 \mathrm{E}-10$ & $4.3824 \mathrm{E}-10$ & $4.3751 \mathrm{E}-10$ \\
\hline 2 & 1.6459E-09 & $1.6414 \mathrm{E}-09$ & $5.0930 \mathrm{E}-10$ & $5.0756 \mathrm{E}-10$ & $4.3717 \mathrm{E}-10$ & $4.3525 \mathrm{E}-10$ \\
\hline 4 & $1.6400 \mathrm{E}-09$ & $1.6377 \mathrm{E}-09$ & $5.0707 \mathrm{E}-10$ & $5.0643 \mathrm{E}-10$ & $4.3479 \mathrm{E}-10$ & $4.3430 \mathrm{E}-10$ \\
\hline 8 & $1.6376 \mathrm{E}-09$ & $1.6376 \mathrm{E}-09$ & $5.0642 \mathrm{E}-10$ & $5.0642 \mathrm{E}-10$ & $4.3430 \mathrm{E}-10$ & $4.3430 \mathrm{E}-10$ \\
\hline 16 & $1.6376 \mathrm{E}-09$ & $1.6376 \mathrm{E}-09$ & $5.0642 \mathrm{E}-10$ & $5.0642 \mathrm{E}-10$ & 4.3430E-10 & $4.3430 \mathrm{E}-10$ \\
\hline Ref. & \multicolumn{2}{|c|}{$1.6023 \mathrm{E}-09$} & \multicolumn{2}{|c|}{$5.0121 \mathrm{E}-10$} & \multicolumn{2}{|c|}{$4.3053 \mathrm{E}-10$} \\
\hline
\end{tabular}

Table 2 Angles of twist at the loaded tips for tubular cross-section beam problems under uniform torsion with constrained warping. Eight 2-node beam elements are used $(N=8)$

\begin{tabular}{cccc}
\hline \hline Cross-sectional elements used & $t / a=0.1$ & $t / a=0.5$ & $t / a=0.999$ \\
\hline 1st order (4-node ele.) & $1.6269 \mathrm{E}-09$ & $4.8751 \mathrm{E}-10$ & $4.0053 \mathrm{E}-10$ \\
2nd order (9-node ele.) & $1.6288 \mathrm{E}-09$ & $4.9997 \mathrm{E}-10$ & $4.3047 \mathrm{E}-10$ \\
3rd order (16-node ele.) & $1.6376 \mathrm{E}-09$ & $5.0642 \mathrm{E}-10$ & $4.3430 \mathrm{E}-10$ \\
\hline Ref. & $1.6023 \mathrm{E}-09$ & $5.0121 \mathrm{E}-10$ & $4.3053 \mathrm{E}-10$ \\
\hline
\end{tabular}


Table 3 Angles of twist at the loaded tips for angle cross-section beam problems under non-uniform torsion with constrained warping. Three 16-node cross-sectional elements are used and $N$ denotes the number of beam elements used

\begin{tabular}{|c|c|c|c|c|c|c|}
\hline \multirow{2}{*}{$N$} & \multicolumn{2}{|c|}{$t / a=0.1$} & \multicolumn{2}{|c|}{$t / a=0.5$} & \multicolumn{2}{|c|}{$t / a=0.999$} \\
\hline & 2-node beam & 3-node beam & 2-node beam & 3-node beam & 2-node beam & 3-node beam \\
\hline 1 & $1.0220 \mathrm{E}-07$ & $1.0065 \mathrm{E}-07$ & $1.0287 \mathrm{E}-09$ & $1.0196 \mathrm{E}-09$ & $2.1845 \mathrm{E}-10$ & $2.1779 \mathrm{E}-10$ \\
\hline 2 & $9.9214 \mathrm{E}-08$ & $9.7687 \mathrm{E}-08$ & 1.0110E-09 & $9.9918 \mathrm{E}-10$ & $2.1716 \mathrm{E}-10$ & $2.1602 \mathrm{E}-10$ \\
\hline 4 & $9.6044 \mathrm{E}-08$ & $9.6469 \mathrm{E}-08$ & $9.8671 \mathrm{E}-10$ & $9.8918 \mathrm{E}-10$ & $2.1479 \mathrm{E}-10$ & $2.1486 \mathrm{E}-10$ \\
\hline 8 & $9.5940 \mathrm{E}-08$ & 9.6073E-08 & 9.8484E-10 & $9.8594 \mathrm{E}-10$ & $2.1437 \mathrm{E}-10$ & $2.1449 \mathrm{E}-10$ \\
\hline 16 & $9.5940 \mathrm{E}-08$ & 9.5973E-08 & $9.8484 \mathrm{E}-10$ & $9.8511 \mathrm{E}-10$ & $2.1437 \mathrm{E}-10$ & $2.1440 \mathrm{E}-10$ \\
\hline Ref. & \multicolumn{2}{|c|}{$9.7160 \mathrm{E}-08$} & \multicolumn{2}{|c|}{$1.0008 \mathrm{E}-09$} & \multicolumn{2}{|c|}{$2.1629 \mathrm{E}-10$} \\
\hline
\end{tabular}

Table 4 Angles of twist at the loaded tips for angle cross-section beam problems under non-uniform torsion with constrained warping. Eight 2-node beam elements are used $(N=8)$

\begin{tabular}{cccc}
\hline \hline Cross-sectional elements used & $t / a=0.1$ & $t / a=0.5$ & $t / a=0.999$ \\
\hline 1st order (4-node ele.) & $9.3925 \mathrm{E}-08$ & $8.5206 \mathrm{E}-10$ & $1.8475 \mathrm{E}-10$ \\
2nd order (9-node ele.) & $9.5601 \mathrm{E}-08$ & $9.5350 \mathrm{E}-10$ & $2.0308 \mathrm{E}-10$ \\
3rd order (16-node ele.) & $9.5940 \mathrm{E}-08$ & $9.8484 \mathrm{E}-10$ & $2.1437 \mathrm{E}-10$ \\
\hline Reference solutions & $9.7160 \mathrm{E}-08$ & $1.0008 \mathrm{E}-09$ & $2.1629 \mathrm{E}-10$ \\
\hline
\end{tabular}

\subsection{Various solid cross-section beam problems}

Let us consider straight beam problems with three different solid cross-sections (trapezoidal, circular and spline cross-sections) in Fig. 11. The beam length is $L=20 \mathrm{~m}$, the boundary condition for free warping are used as in Eq. (21) and the torsional moment $M_{x}=1.0 \mathrm{~N} \cdot \mathrm{m}$ is applied at the center of twist at $x=L$ as shown in Fig. 5(a). A single cross-sectional element is used for the trapezoidal and circular cross-sections and seven cross-sectional elements are used for the spline cross-section. In the beam length direction, a single 2-node beam element is used. Reference

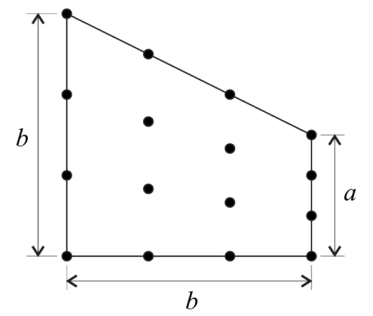

(a)

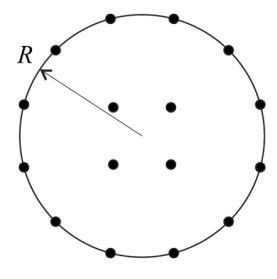

(b)

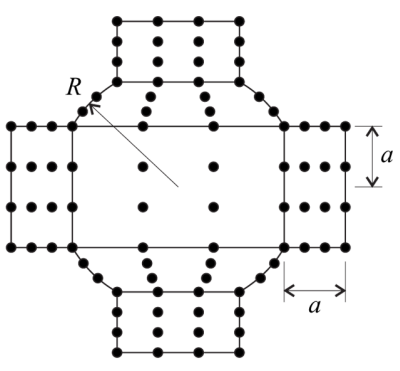

(c)

Fig. 11 Cross-sectional discretizations of various solid cross-sections modeled on the $y z$-plane. (a) Trapezoidal cross-section modeled by a single 16-node cross-sectional element $(a=2, b=4)$, (b) circular cross-section modeled by a single 16-node cross-sectional element $(R=2)$, (c) solid crosssection with four splines modeled by seven 16-node cross-sectional elements $(R=2, a=1)$ 
Table 5 Angles of twist at the loaded tip for various solid cross-section beam problems

\begin{tabular}{ccccc}
\hline \hline Cross-section & $\begin{array}{c}\text { 1st order } \\
\text { (4-node } \\
\text { cross-sectional el.) }\end{array}$ & $\begin{array}{c}\text { 2nd order } \\
\text { (9-node } \\
\text { cross-sectional ele.) }\end{array}$ & $\begin{array}{c}\text { 3rd order } \\
\text { (16-node } \\
\text { cross-sectional ele.) }\end{array}$ & Reference solutions \\
\hline Trapezoidal & $8.3158 \mathrm{E}-12$ & $9.1001 \mathrm{E}-12$ & $1.0845 \mathrm{E}-11$ & $1.0632 \mathrm{E}-11$ \\
Circular & $1.8750 \mathrm{E}-11$ & $8.1482 \mathrm{E}-12$ & $7.9401 \mathrm{E}-12$ & $7.9578 \mathrm{E}-12$ \\
Spline & $4.2236 \mathrm{E}-12$ & $4.3103 \mathrm{E}-12$ & $4.6444 \mathrm{E}-12$ & $4.2890 \mathrm{E}-12$ \\
\hline
\end{tabular}
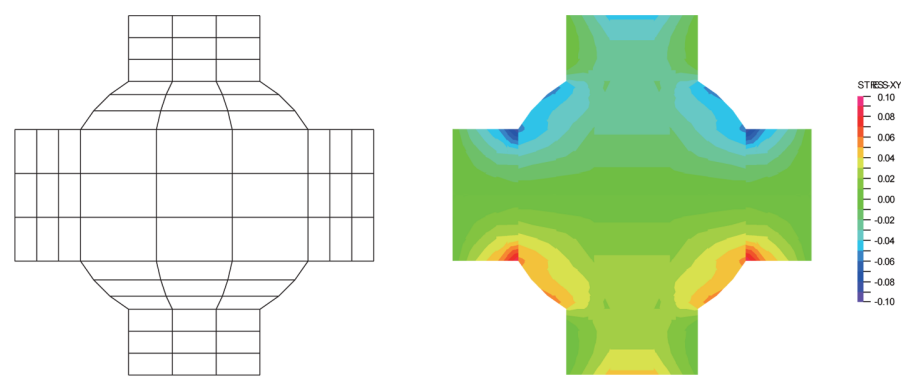

(a)
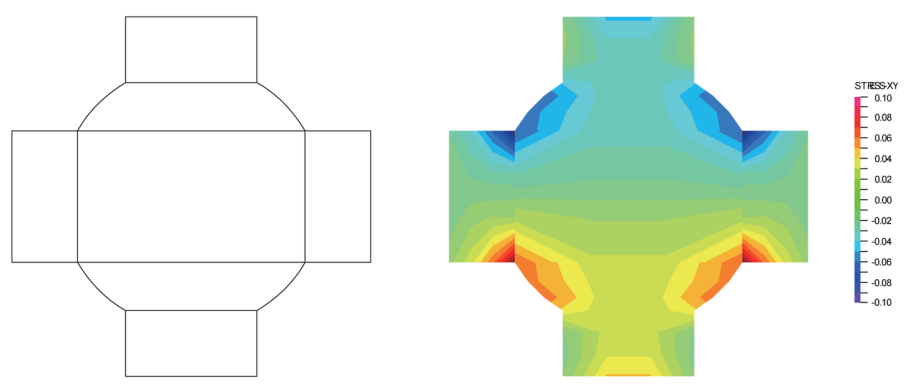

(b)

Fig. 12 Distributions of transverse shear stress- $x y$ in the solid cross-section with four splines. (Left: crosssectional meshes used, Right: stress distribution) (a) solid element solution calculated by 630 27-node solid elements. 63 and 10 solid elements are used on cross-sections and in beam length, respectively. (b) beam element solution calculated by three beam elements. Seven 16-node cross-sectional elements are used.

solutions are obtained from (Roark 1965).

The results in Table 5 suggest that the single 16-node cross-sectional element gives good accuracy for the trapezoidal and circular sections. For the cross-section with four splines, more cross-sectional elements will give better results. To this point, we overall conclude that the 16-node cross-sectional element with the 3rd order interpolation function is effective in general cross-section cases including thin- and thick-walled cross-sections.

Additionally, we analyze the spline cross-section beam problem with constrained warping by using three beam elements with seven 16-node cross-sectional elements. The boundary condition in Eq. (22) is used. The results are compared with the solutions of the solid element model with 630 
27-node solid elements. Fig. 12 shows the distributions of transverse shear stress-xy on the crosssectional plane at $x=L / 2$. The angles of twist calculated at the loaded tips are $4.6051 \mathrm{E}-12$ and 4.5968E-12 for the solid and beam element models, respectively.

\subsection{Curved wide flange cross-section beam problems}

We consider the curved wide flange cross-section beam problems shown in Fig. 13. The geometry of the beam is a quarter arc. The boundary conditions at $\phi=0^{\circ}$ are $u=v=w=\theta_{x}=\theta_{y}=\theta_{z}=0$ for free warping and $u=v=w=\theta_{x}=\theta_{y}=\theta_{z}=\alpha=0$ for constrained warping. The beam is subjected to the torsional moment $M_{y}=1.0 \mathrm{~N} \cdot \mathrm{m}$ at the tip $\left(\phi=90^{\circ}\right)$. We use seven 16-node cross-sectional elements for the wide flange cross-section as shown in Fig. 13(a).

Since the beam problems have thin-walled cross-sections, shell finite element solutions are used as references. Fig. 13(b) shows the shell finite element model including the mesh, the boundary conditions for free and constrained warping and the external loading equivalent to the beam problem. For each plate, ten MITC9 shell elements are used along the beam length, see (Bucalem
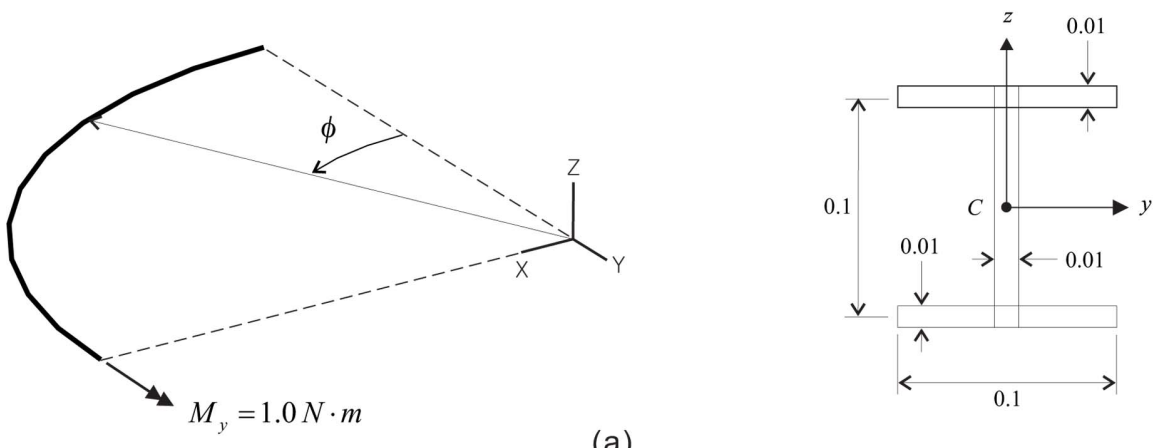

(a)
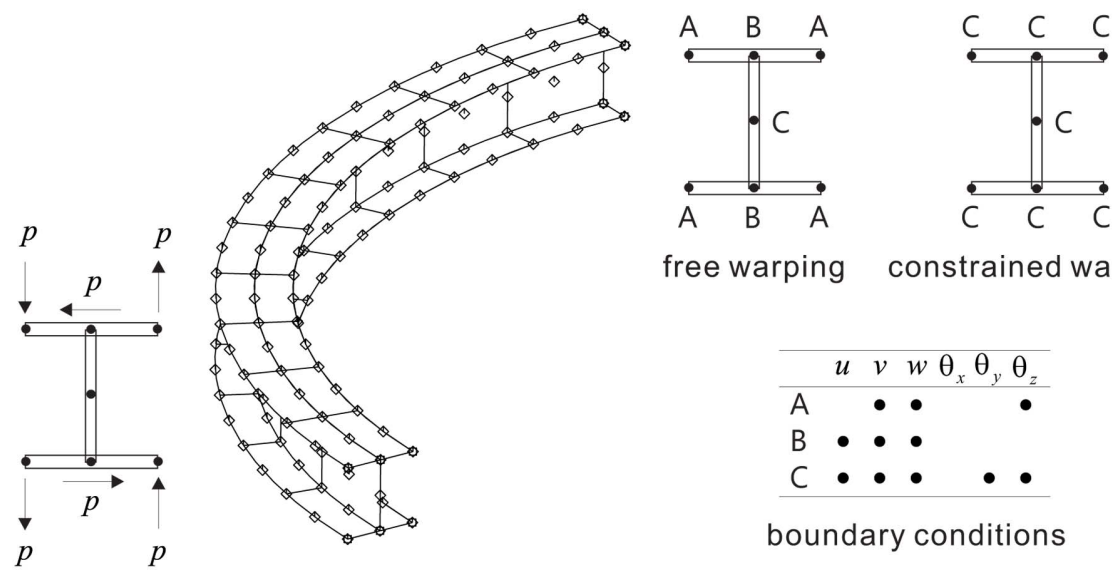

free warping constrained warping

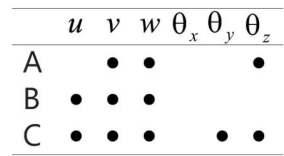

boundary conditions

(b)

Fig. 13 Curved wide flange cross-section beam problems. (a) geometry of the curved wide flange crosssection beam problem and cross-sectional mesh used, (b) shell finite element models used 

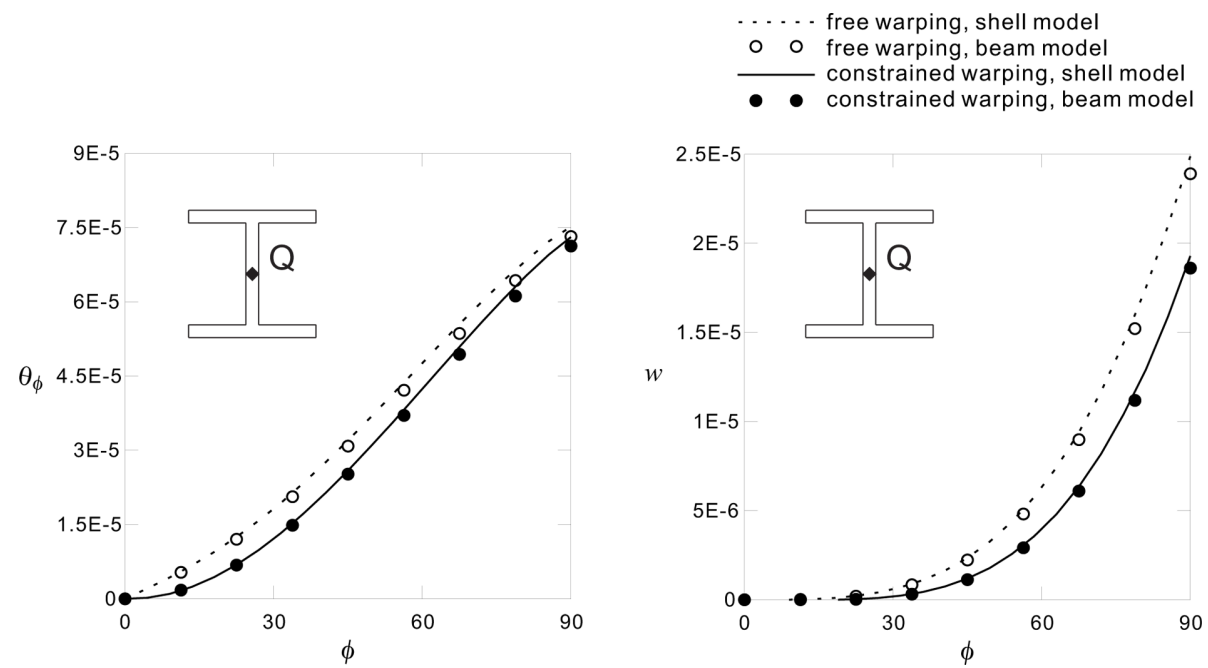

Fig. 14 Angle of twist and displacement $w$ along the beam length in the curved wide flange cross-section beam problems

Table 6 Angles of twist and displacement $w$ at the loaded tip for the curved wide flange cross-section beam problems according to the warping direction used. Four 2-node beam elements $(N=4)$ and 16-node cross-sectional elements are used

\begin{tabular}{cccccc}
\hline \hline \multirow{2}{*}{ Warping direction } & \multicolumn{2}{c}{ Free warping } & & \multicolumn{2}{c}{ Constrained warping } \\
\cline { 2 - 3 } \cline { 5 - 6 } & Angle of twist & $w$ & & Angle of twist & $w$ \\
\hline Fig. 3(a) & $7.5199 \mathrm{E}-05$ & $2.3273 \mathrm{E}-05$ & & $7.3136 \mathrm{E}-05$ & $1.7909 \mathrm{E}-05$ \\
Fig. 3(c) & $7.2384 \mathrm{E}-05$ & $2.2232 \mathrm{E}-05$ & & $7.0503 \mathrm{E}-05$ & $1.7233 \mathrm{E}-05$ \\
\hline Ref. & $7.5220 \mathrm{E}-05$ & $2.4925 \mathrm{E}-05$ & & $7.3547 \mathrm{E}-05$ & $1.9020 \mathrm{E}-05$ \\
\hline
\end{tabular}

and Bathe 1993, Bathe et al. 2003). In the figure, the point load is $p=10 / 3 N$ and A, B and C denote the boundary conditions used. Note that to obtain a proper beam model for free warping, special attention needs to be carefully given. The angles of twist for the reference solutions are obtained from the rotation degrees of freedom of the shell elements.

Fig. 14 shows the distributions of the angle of twist and displacement $w$ along the beam length corresponding to the point $\mathrm{Q}$ when four 3-node beam elements are used. Although the torsional moment is applied at the free tip, it is very hard to accurately calculate the response of this beam problem without considering the warping effect coupled with bending, shearing and stretching. Note that in the thin-walled cross-section beam problems, the results calculated by 4-node or 9-node cross-sectional elements are almost the same.

In the beam problems, we test the effect of the warping direction used. Table 6 shows the numerical results when the warping directions in Figs. 3(a) and (c) are used. To clearly investigate the effect, four 2-node elements are used. The warping direction in Fig. 3(a) gives better results. However, when the number of the beam elements used increases or higher order beam elements are used, the difference becomes smaller. 
Table 7 Angles of twist and displacement $w$ at the loaded tip for the curved wide flange cross-section beam problems with constrained warping according to $D / R$. 16-node cross-sectional elements are used and $N$ denotes the number of beam elements used

\begin{tabular}{|c|c|c|c|c|c|c|}
\hline \multirow{2}{*}{$D / R$} & \multicolumn{3}{|c|}{ Angle of twist } & \multicolumn{3}{|c|}{$w$} \\
\hline & $N=4$ & $N=8$ & Ref. & $N=4$ & $N=8$ & Ref. \\
\hline 0.1 & 7.1272E-05 & $7.1265 \mathrm{E}-05$ & 7.3547E-05 & $1.8613 \mathrm{E}-05$ & $1.8599 \mathrm{E}-05$ & $1.9020 \mathrm{E}-05$ \\
\hline 0.2 & $1.5101 \mathrm{E}-05$ & $1.5102 \mathrm{E}-05$ & $1.8024 \mathrm{E}-05$ & 4.0321E-06 & $4.0296 \mathrm{E}-06$ & 4.2771E-06 \\
\hline 0.5 & $2.4656 \mathrm{E}-07$ & $2.4659 \mathrm{E}-07$ & $3.9216 \mathrm{E}-06$ & $6.1726 \mathrm{E}-08$ & $6.1694 \mathrm{E}-08$ & $6.1724 \mathrm{E}-08$ \\
\hline
\end{tabular}

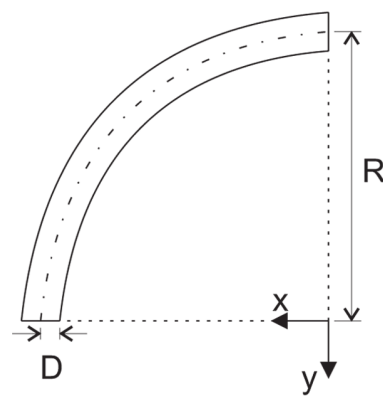

(a)

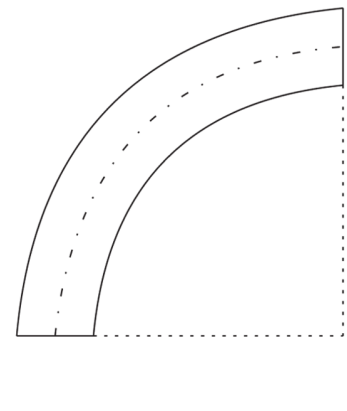

(b)

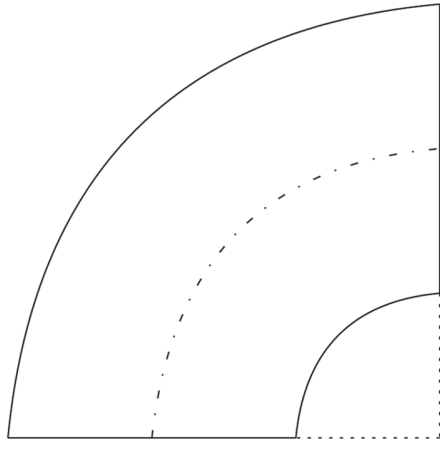

(c)

Fig. 15 Curved wide flange cross-section beam problems with three different $D / R$. (a) $D / R=0.1$, (b) $D / R=$ 0.2, (c) $D / R=0.5$

Table 7 shows the numerical results depending on $D / R$ defined in Fig. 15. As $D / R$ increases (that is, the beam becomes deeper), the angles of twist calculated shows a bigger difference from the reference solutions because the effect of local deformation that cannot be captured by our beam element becomes more dominant.

\subsection{Varying wide flange cross-section beam problems}

Consider here the straight wide flange beam with a varying cross-section shown in Fig. 16. The boundary conditions are at $x=0$, are $u=v=w=\theta_{x}=\theta_{y}=\theta_{z}=0$ for free warping and $u=v=w$ $=\theta_{x}=\theta_{y}=\theta_{z}=\alpha=0$ for constrained warping. The beam dimensions are linearly decreasing from $x=0$ to $x=1.0$ except for the plate thicknesses that remain constant. A torsional moment $M_{x}=1.0 \mathrm{~N} \cdot \mathrm{m}$ is applied at tip $(x=1.0)$. For the beam model, seven 16-node cross-sectional elements for the wide flange cross-section are used as shown in Fig. 16(a).

For the shell finite element model to calculate reference solutions, see Fig. 16(b), $p=20 / 3 N$ and the boundary conditions for the free and constrained warping cases are the same as in the curved wide flange cross-section beam problems. The angles of twist for the reference solutions are obtained from the rotation degrees of freedom of the shell elements. 

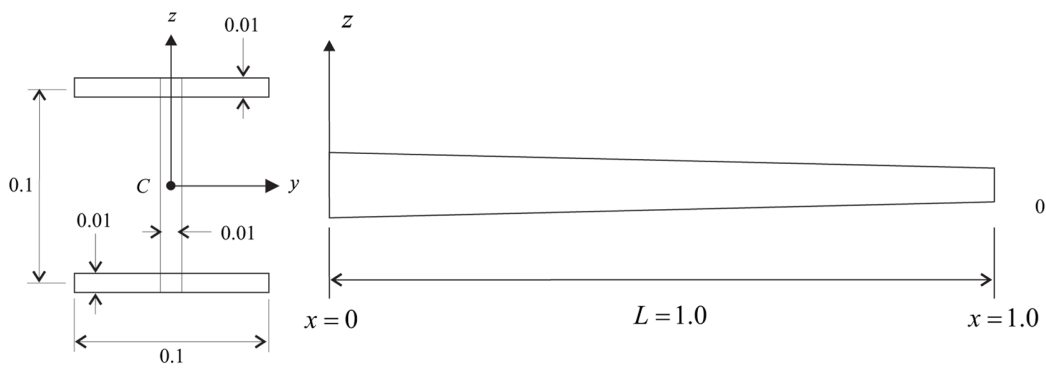

(a)

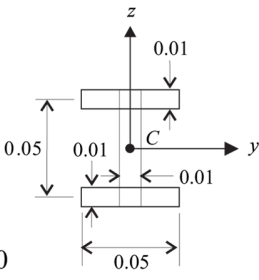

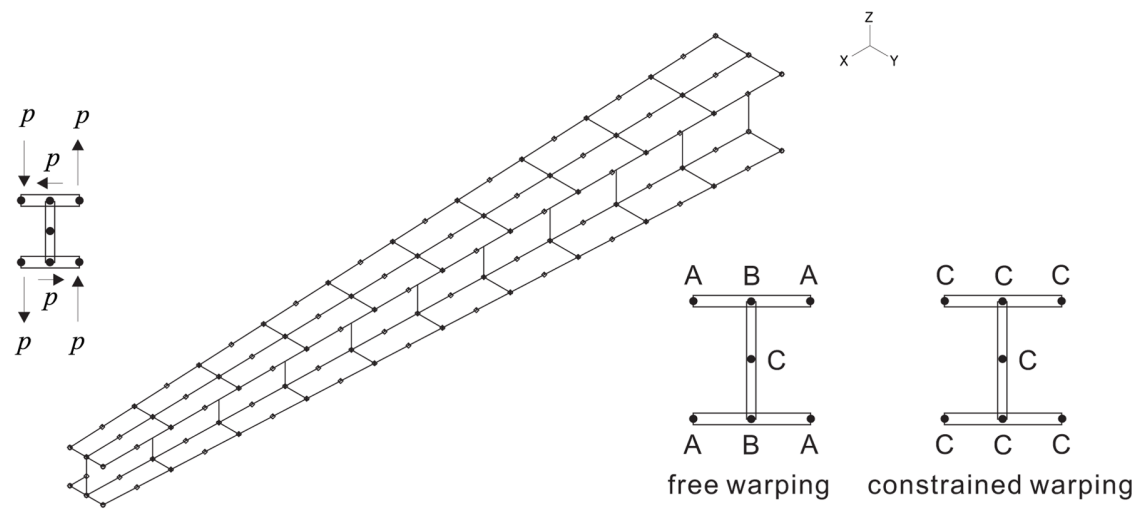

(b)

Fig. 16 Varying wide flange cross-section beam problems. (a) geometry of the varying wide flange crosssection beam problem and cross-sectional mesh used, (b) shell finite element models used
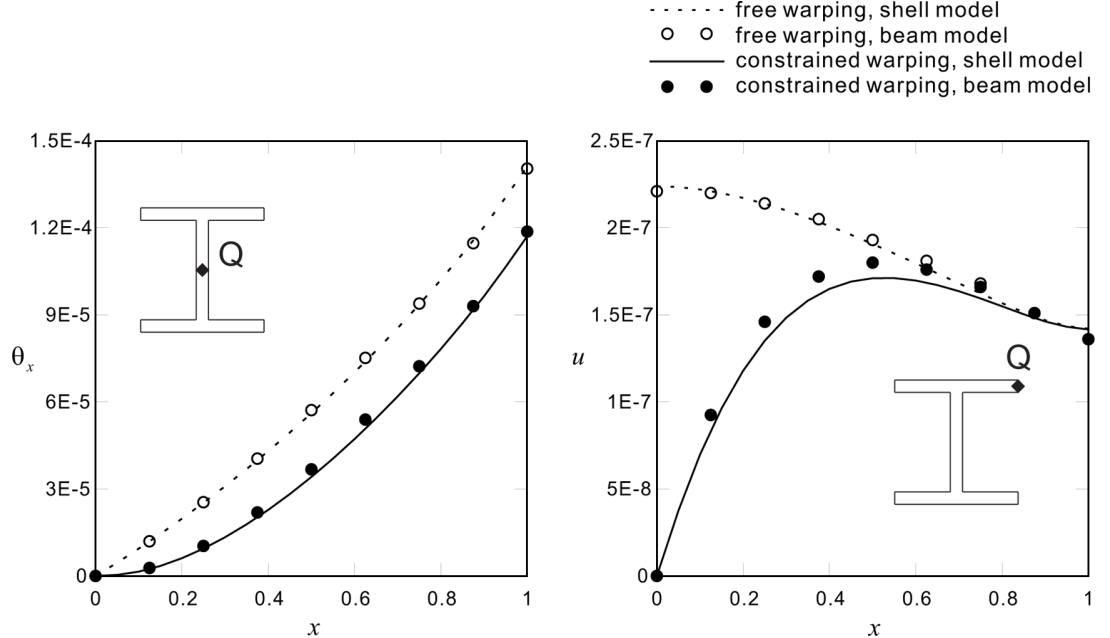

Fig. 17 Angle of twist and displacement $u$ along the beam length in the varying wide flange cross-section beam problems 
Table 8 Angles of twist at the loaded tips for the varying wide flange cross-section beam problems under uniform torsion. Four 3-node beam elements are used $(N=4)$

\begin{tabular}{cccccc}
\hline \hline \multirow{2}{*}{$\tan 0.5 \theta$} & \multicolumn{2}{c}{ Free warping } & & \multicolumn{2}{c}{ Constrained warping } \\
\cline { 2 - 3 } \cline { 5 - 6 } & Present study & Ref. & & Present study & Ref. \\
\hline 0.025 & $1.3771 \mathrm{E}-04$ & $1.40641 \mathrm{E}-04$ & & $1.1454 \mathrm{E}-04$ & $1.16549 \mathrm{E}-04$ \\
0.095 & $8.5779 \mathrm{E}-05$ & $8.74165 \mathrm{E}-05$ & & $5.9483 \mathrm{E}-05$ & $6.01527 \mathrm{E}-05$ \\
0.225 & $3.8829 \mathrm{E}-05$ & $3.86123 \mathrm{E}-05$ & & $2.0123 \mathrm{E}-05$ & $1.91705 \mathrm{E}-05$ \\
0.475 & $2.0648 \mathrm{E}-05$ & $1.87122 \mathrm{E}-05$ & & $8.2445 \mathrm{E}-06$ & $7.11663 \mathrm{E}-06$ \\
\hline
\end{tabular}

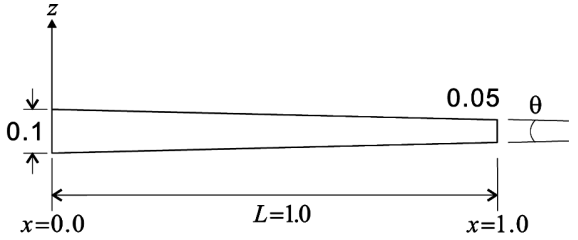

(a)

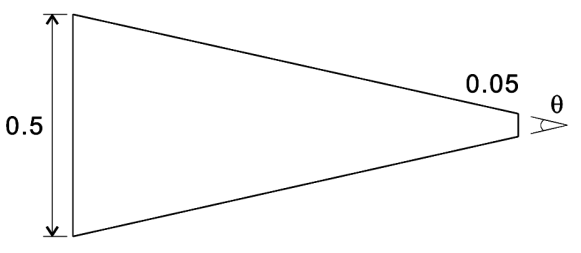

(c)

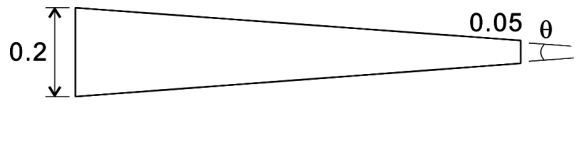

(b)

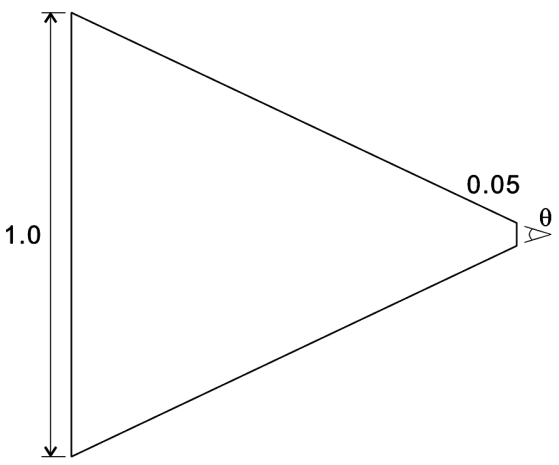

(d)

Fig. 18 Varying wide flange cross-section beam problems with four different tapering degrees. (a) $\tan 0.5 \theta=0.025 \quad(\theta=2.864$ deg. $), \quad(b) \tan 0.5 \theta=0.095 \quad(\theta=10.854$ deg. $), \quad(c) \tan 0.5 \theta=0.225(\theta=$ 25.361 deg.), (d) $\tan 0.5 \theta=0.475$ ( $\theta=50.815$ deg. $)$

Fig. 17 shows the distributions of the displacements (the angles of twist and the warping displacement) corresponding to the point $\mathrm{Q}$ when four 3-node beam elements are used. Table 8 presents the angles of twist calculated for four different tapering degrees defined by $\tan 0.5 \theta$ in Fig. 18. Until the tapering degree $\tan 0.5 \theta=0.225$, the numerical results show reasonable accuracy compared with the reference solutions. However, when the tapering degree is extremely high as in the case of $\tan 0.5 \theta=0.475$, the difference between the beam and shell solutions are large although more beam finite elements are used.

In Fig. 19, we present the displacement distribution when an eccentric lateral force $P_{y}=1.0 \mathrm{~N}$ is applied at the free tip $(x=1.0)$ in order to result in a flexure-torsion problem as shown in Fig. 19(a), and warping is constrained at the fixed end and $\tan 0.5 \theta=0.025$. 


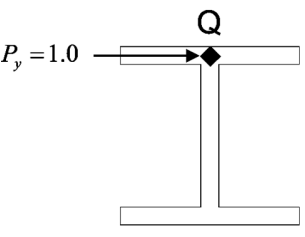

(a)
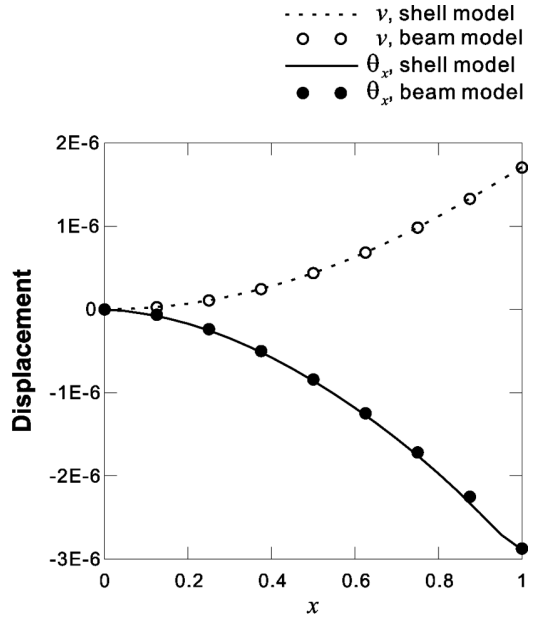

(b)

Fig. 19 Displacement $v$ and angle of twist along the beam length in varying wide flange cross-section beam problems with an eccentric end tip load $(\tan 0.5 \theta=0.025)$

\subsection{Wind turbine blade problem}

We consider the wind turbine blade problem shown in Fig. 20(a). The geometry of the blade is described by the equations in Appendix with design parameters $L=35 \mathrm{~m}, c_{0}=8.395 \mathrm{~m}, c_{L}=3.425 \mathrm{~m}$, $\beta_{0}=29$ deg., $\beta_{L}=3.6$ deg., $p=0.4, m=0.04, h=0.125$. Three stiffeners are positioned at $s=0.2,0.5$ and 0.75 along the blade length. The airfoil surfaces and stiffeners have thickness $t=0.05 \mathrm{~m}$.

The boundary condition at $x=0$ is $u=v=w=\theta_{x}=\theta_{y}=\theta_{z}=\alpha=0$, that is, warping is also
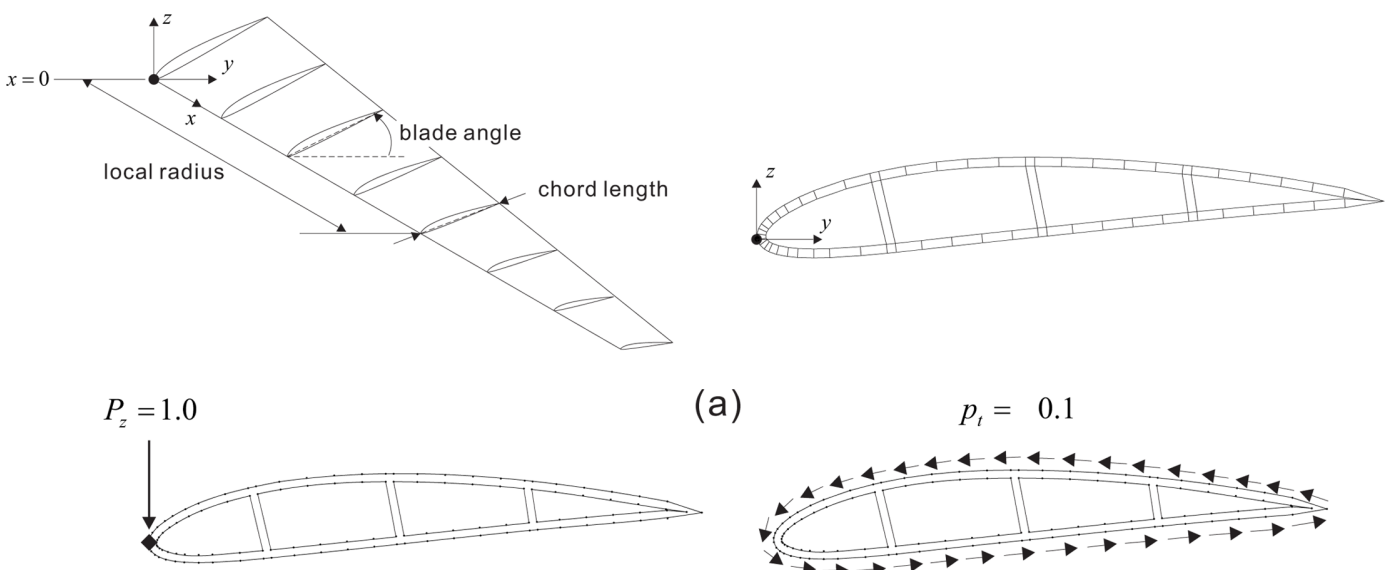

(a)

$p_{t}=0.1$

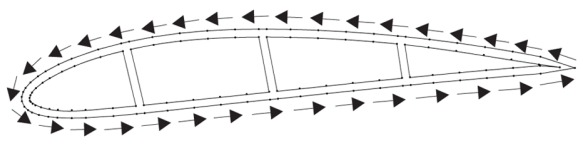

(b)

Fig. 20 Wind turbine blade problems. (a) geometry of the wind turbine blade with a NACA airfoil and crosssectional mesh used, (b) loading conditions. Left: flexural-torsion case, Right: pure torsion case (resultant moment: $M_{x}=2.0 \mathrm{~N} \cdot \mathrm{m}$ ) 

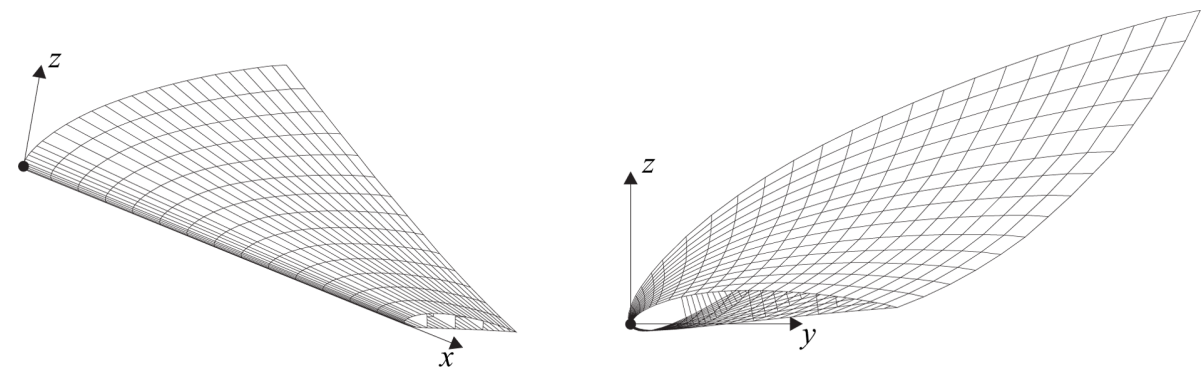

Fig. 21 Shell finite element model for the wind turbine blade problems
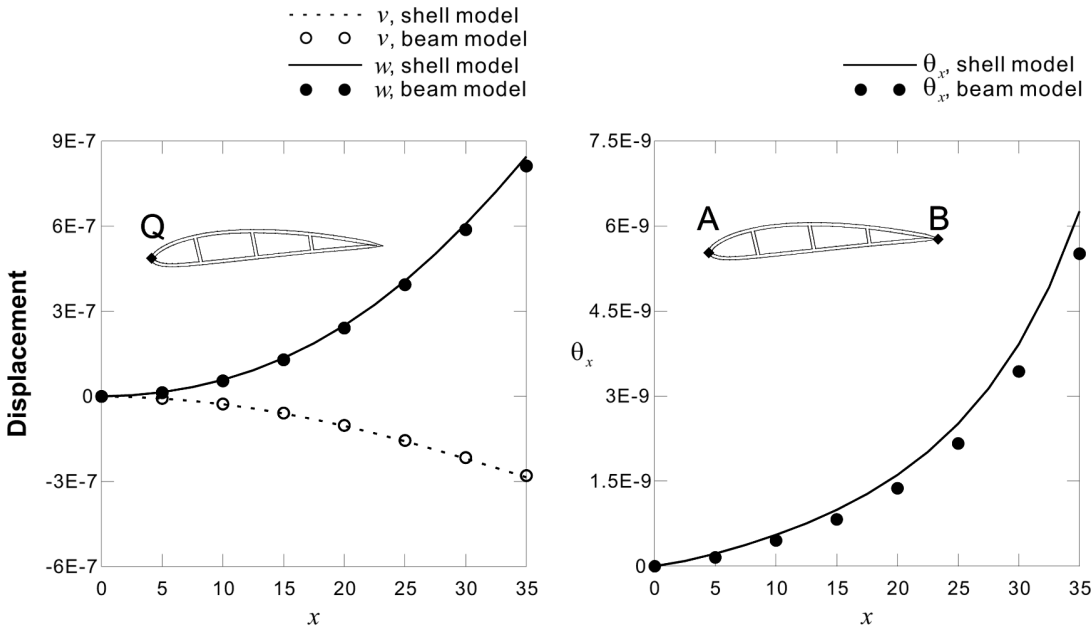

(a)

(b)

Fig. 22 Displacements $v, w$ and the angle of twist along the beam length in the wind turbine blade problems. (a) flexural-torsion case. The displacements are given at point $\mathbf{Q}$. (b) pure torsion case. For the shell model, the angle of twist is evaluated from the relative displacements between two points $\mathbf{A}$ and $\mathbf{B}$ because the distribution of the angle is not uniform on the cross-section due to the effect of local deformation, but the angle of twist for the beam model is given at point $\mathbf{A}$

constrained. Fig. 20(b) shows two different loading conditions on the cross-section at $x=L$ : a transverse point load $\left(P_{z}=1.0 \mathrm{~N}\right)$ to result in flexural-torsion behavior and a uniformly distributed load $\left(p_{t}=1.0 \mathrm{~N} / \mathrm{m}\right)$ around the airfoil surface to result in pure torsion.

To analyze the wind turbine blade problem using the beam finite elements, we use seven beam finite elements and sixty-one 4-node cross-sectional elements for the airfoil cross-section as in Fig. 20(a). The number of total degrees of freedom used is 56 .

Using the MITC9 shell finite elements, reference solutions are calculated, see Fig. 21. All the degrees of freedom are fixed at $x=0$. We use 770 shell elements and 4680 degrees of freedom for the shell model.

Figs. 22(a) and (b) show the numerical results for the flexural-torsion case and pure torsion cases, respectively. Although, for the beam model, we use only 1.2 percent of the degrees of freedom of the shell models, the beam model solutions exhibit excellent accuracy compared with the shell model solutions. 


\section{Conclusions}

In this paper we proposed a continuum mechanics based beam finite element with warping displacements. We presented the general formulation including how the geometry and displacements are defined and how warping displacements are efficiently accounted for. The novel features of the beam element are the simple and straightforward formulation, the inclusion of fully coupled warping effects, the ability of handling complicated geometry and only one additional degree of freedom at each beam node.

The various numerical results showed the effectiveness of the beam elements. Especially, excellent modeling capabilities and solution accuracy of the proposed beam element were observed. Regarding the beam problems considered, we have made the following observations:

- In order to correctly predict the torsional behaviors of beams with general cross-sections, the use of 16-node cross-sectional elements is effective. However, thin-walled cross-section beam problems, 4-node or 9-node cross-sectional elements can be enough.

- In most of the analyses presented, two 2-node beam elements and one 3-node beam element give solutions accurate enough for engineering practices.

- The use of the warping directions normal to cross-sections at beam nodes is recommended for accurate analyses of general curved beam problems.

While we assumed in this paper linear elastic behavior, an important asset of the beam formulation is that it can be directly extended to efficient geometric and material nonlinear analyses, see (Lee and McClure 2006, Lee and McClure 2007, Pi et al. 2005), and this will be described in future works. The beam element can also consider other patterns of beam deformation by properly enriching the basic displacement interpolation in Eq. (6).

\section{Acknowledgements}

This work was supported by the Human Resources Development (No.20114030200040) of the Korea Institute of Energy Technology Evaluation and Planning (KETEP) grant funded by the Korea government Ministry of Knowledge Economy.

\section{References}

Abbott, I.H., Von Doenhoff, A.E. and Stivers, L.S. (1945), Summary of Airfoil Data, NACA report 824, Langley Field.

Bathe, K.J. (1996), Finite Element Procedures, Prentice Hall, New York.

Bathe, K.J. and Chaudhary, A. (1982), "On the displacement formulation of torsion of shafts with rectangular cross-section", Int. J. Numer. Meth. Eng., 18, 1565-1568.

Bathe, K.J., Lee, P.S. and Hiller, J.F. (2003), "Towards improving the MITC9 shell element”, Comput. Struct., 81, 477-489.

Bucalem, M.L. and Bathe, K.J. (1993), "Higher-order MITC general shell elements", Int. J. Numer. Meth. Eng., 36, 3729-3754.

Chapelle, D. and Bathe, K.J. (2003), The Finite Element Analysis of Shells - Fundamentals, Springer.

Dutta, A. and White, D.W. (1992), "Large displacement formulation of a three-dimensional beam element with 
cross-sectional warping", Comput. Struct., 45, 9-24.

Dvorkin, E.N. and Bathe, K.J. (1984), "A continuum mechanics based four-node shell element for general nonlinear analysis", Eng. Comput., 1, 77-88.

Gjelsvik, A. (1981), The Theory of Thin Walled Bars, Wiley, New York.

Gonçalves, R., Ritto-Corrêa, M. and Camotim, D. (2010), "A new approach to the calculation of cross-section deformation modes in the framework of generalized beam theory", Comput., Mech., 46, 759-781.

Gruttmann, F., Sauer, R. and Wagner, W. (1999), "Shear stresses in prismatic beams with arbitrary crosssections", Int. J. Numer. Meth. Eng., 45, 865-889.

Lee, P.S. and Bathe, K.J. (2004), "Development of MITC isotropic triangular shell finite elements", Comput. Struct., 82, 945-962.

Lee, P.S. and Bathe, K.J. (2005), "Insight into finite element shell discretizations by use of the basic shell mathematical model", Comput. Struct., 83, 69-90.

Lee, P.S. and McClure, G. (2006), "A general 3D L-section beam finite element for elastoplastic large deformation analysis", Comput. Struct., 84, 215-229.

Lee, P.S. and McClure, G. (2007), "Elastoplastic large deformation analysis of a lattice steel tower structure and comparison with full-scale tests", Int. J. Constr. Steel Res., 63, 709-717.

Lee, P.S. and Noh, H.C. (2010), "Inelastic buckling behavior of steel members under reversed cyclic loading", Eng. Struct., 32, 2579-2595.

Lee, P.S., Noh, H.C. and Choi, C.K. (2008), "Geometry-dependent MITC method for a 2-node iso-beam element”, Struct. Eng. Mech., 29(2), 203-221.

Lee, S.W. and Kim, Y.H. (1987), "A new approach to the finite element modelling of beams with warping effect”, Int. J. Numer. Meth. Eng., 24, 2327-2341.

Pi, Y.L., Bradford, M.A. and Uy, B. (2005), "Nonlinear analysis of members curved in space with warping and Wagner effects", Int. J. Solids Struct., 42, 3147-3169.

Prokiæ, A. (1996), "New warping function for thin-walled beams. I: theory", J. Struct. Eng., 122, 1437-1442.

Roark, R.J. (1965), Formulas for Stress and Strain, McGraw Hill, New York.

Timoshenko, S.P. and Goodier, J.N. (1970), Theory of Elasticity, McGraw Hill, New York.

Vlasov, V.Z. (1961), Thin-walled Elastic Beams, Israel Program for Scientific Translations, Jerusalem.

Živkovic, M., Kojić, M., Slavković, R. and Grujović, N. (2001), "A general beam finite element with deformable cross-section", Comput. Meth. Appl. Mech. Eng., 190, 2651-2680. 


\section{Appendix: Geometry of the wind turbine blade}

We here give the equations to define the geometry of the wind turbine blade with a NACA airfoil. The equations for the airfoil shape are given by the NACA four-digit series (Abbott et al. 1945).

The chord length $c$ and blade angle $\beta$ (deg.) vary depending on the local radius $x$

$$
c=c_{0}-\left(c_{0}-c_{L}\right) \frac{x}{L} \quad \text { and } \quad \beta=\beta_{0}-\left(\beta_{0}-\beta_{L}\right) \frac{x}{L}
$$

in which $L$ is the blade length, $c_{0}$ and $c_{L}$ are the chord lengths at $x=0$ and $x=L$, respectively, and $\beta_{0}$ and $\beta_{L}$ are the blade angles at $x=0$ and $x=L$, respectively.

The height distribution of the airfoil with unit length is

$$
z_{t}=\frac{h}{0.2}\left(0.2629 \sqrt{s}-0.1260 s-0.3516 s^{2}+0.2843 s^{3}-0.1015 s^{4}\right)
$$

where $h$ is the maximum height of the airfoil.

The mean camber line is defined by the maximum camber $m$ and its position $p$

$$
z_{c}= \begin{cases}m \frac{s}{p}(2 p-s), & \text { for } 0 \leq s \leq p \\ m \frac{1-s}{(1-p)^{2}}(1+s-2 p), & \text { for } p \leq s \leq 1\end{cases}
$$

The coordinates for the airfoil upper mid-surface $\left(y_{U}, z_{U}\right)$ and lower mid-surface $\left(y_{L}, z_{L}\right)$ are obtained by

$$
\left[\begin{array}{l}
y_{U} \\
z_{U}
\end{array}\right]=c\left[\begin{array}{cc}
\cos \beta & -\sin \beta \\
\sin \beta & \cos \beta
\end{array}\right]\left[\begin{array}{c}
s-z_{t} \sin \theta \\
z_{c}+z_{t} \cos \theta
\end{array}\right], \quad\left[\begin{array}{l}
y_{L} \\
z_{L}
\end{array}\right]=c\left[\begin{array}{cc}
\cos \beta & -\sin \beta \\
\sin \beta & \cos \beta
\end{array}\right]\left[\begin{array}{l}
s+z_{t} \sin \theta \\
z_{c}-z_{t} \cos \theta
\end{array}\right]
$$

with $\theta=\arctan \left(\frac{d z_{c}}{d s}\right)$ 